\title{
Porosity Reduction in the High-Speed Processing of Glass-Fiber Composites by Resin Transfer Molding (RTM)
}

\author{
HARry J. BArraza ${ }^{\mathrm{a}}$, Youssef K. HAMidi ${ }^{\mathrm{b}}$, LeVEnT AKtas $^{\mathrm{b}}$, \\ EdGar A. O'REAR ${ }^{\mathrm{a}}$ AND M. C. Altan ${ }^{\mathrm{b}, *}$ \\ ${ }^{a}$ School of Chemical Engineering and Materials Science \\ ${ }^{b}$ School of Aerospace and Mechanical Engineering \\ University of Oklahoma, Norman, OK 73019, USA \\ (Received November 22, 2002) \\ (Revised July 1, 2003)
}

\begin{abstract}
High-speed processing is essential to achieve lower production cost in the fabrication of fiber-reinforced composites with the current liquid molding practices. A major consequence of increasing the resin injection velocity is the formation of defects such as voids and dry regions that decrease the load-bearing capability of the composite. Void formation mechanisms and analytical predictions of the detrimental effect of porosity on the structural integrity of molded parts have been studied extensively. In contrast, knowledge of void removal strategies is very limited. In this investigation, various postfill pressure levels were applied to disk-shaped random-mat glass/epoxy parts molded at high volumetric flow rates as a method to reduce their voidage content. Quantitative image analysis over cross-sections cut from these composites revealed that significant changes in porosity concentration take place with the postfill pressure. For instance, overall void content dropped more than $70 \%$ with the application of a postfill pressure as low as $300 \mathrm{kPa}$. Other important void morphometry characteristics such as void shape, size, and spatial distribution could also be manipulated by this method. As the packing pressure increases, large voids gradually disappear, and at the same time, the small circular voids are mobilized towards radial locations near the vents. In addition to this spatial voidage gradient in the radial direction, voidage gradient also exists through the specimen thickness. It seems that higher front velocities promote the appearance of secondary flow phenomena inside the mold cavity (e.g. microfountain flow), which may explain why more voids tend to concentrate at the surface of the specimen irrespective of the postfill pressure level reached inside the mold.
\end{abstract}

KEY WORDS: resin transfer molding, high-speed injection, void content, postfill pressure.

*Author to whom correspondence should be addressed. E-mail: altan@ou.edu 


\section{INTRODUCTION}

$\mathbf{L}$ IQUID MOLDING TECHNOLOGIES such as Resin Transfer Molding (RTM), Vacuum Assisted Resin Transfer Molding (VARTM) and Resin Infusion, among others, are often regarded by composite manufacturers as excellent alternatives to current autoclave-cured laminated composites. In general, these liquid molding processes have lower operational costs and higher production rates that make them particularly attractive for high-throughput industries such as automotive and consumer products. Some authors even predict that high-speed liquid molding will be the mainstream fabrication process for aerospace parts in the next decade [1,2]. In resin transfer molding an uncured liquid resin is mechanically injected into a mold containing an inorganic porous or fibrous reinforcement. The resin is expected to displace the air inside the mold, quickly filling up all empty spaces within the preform. In the case of fibrous reinforcements, the network of channels for the resin flow is not uniform and contains a wide distribution of intertow and intratow channel spacing as well as a broad range of channel orientations (e.g. random-fiber preforms). Such heterogeneous microstructure forms high and low permeability zones within the reinforcement; and at the same time, creates an imbalance between viscous and surface forces during the flow of the polymeric mixture. Permeability variations at the flow front, coupled with nonisothermal effects coming from the curing kinetics, are responsible for transient phenomena that decrease impregnation quality and ease of spreading of the resin through the inorganic preform, thus leading to macroflow- and microflow-driven defects like dry spots and voids. In addition, voids can also originate from nucleation and growth of either dissolved gases or low molecular weight by-products formed during the crosslinking reaction [3]. High voidage is one of the major factors entailing a severe degradation of mechanical properties in composites [4]. It has been reported that reductions between 2 and $10 \mathrm{MPa}$ in the interlaminar shear strength (ILSS) may occur within a composite part with as low as a $1 \%$ increase in void content [5].

Previous investigations have revealed that void formation during RTM depends primarily on process-related factors such as capillary number, contact angle, and orientation of fibers relative to the flow direction [6]. Patel and Lee [7] put forward a simple unidirectional model that predicts void formation as the result of the extensive fingering at the flow front (e.g. lead-lag behavior). According to these authors, the lead-lag appears when either the intertow or primary flow predominates over the flow between fiber interstices (e.g. capillary flow), and vice versa. The manifestation of flow front lead-lag is directly related to the impregnation rates. For instance, at low filling velocity, the flow inside the fiber tows or intratow flow is dominant. High capillary pressures cause the microflow within the narrow channels formed by two parallel fibers inside a tow to move ahead of the macroflow, which takes place in the intertow spacing. At high flow velocity the situation is reversed: the macroflow moves ahead of the microflow. In this latter case, the capillary effect is very small compared to the externally applied pressure, and therefore the viscous forces dominate the filling pattern [8]. In addition to molding speed, other factors are known to change the lead-lag intensity and ultimately the quantity and distribution of voids inside a molded part. One such variable is the injection mode. When the liquid injection is carried out at a constant volume flow rate, the front lead-lag should not depend on the distance from the inlet for a constant cross-section, one-dimensional flow. Consequently, the void fraction along the composite should not vary much with respect to the inlet distance (i.e. only if void compression 
and advection are assumed negligible). In contrast, when the impregnation is performed at constant inlet pressure the flow rates would decrease with time, and hence, the flow front lead-lag would change as the flow progresses. In this latter case, a spatially nonuniform void distribution is expected, with increasing number of voids present towards the exits [7].

During resin impregnation, and up until the gelling process begins, the entrapped voids are in a transient state where changes in size, shape, and location can be easily induced by applying external driving forces. Several authors [6-10] have studied this particular phenomenon and suggested mechanisms for void mobilization and porosity reduction in RTM composite parts. Lundström [10], for example, found that a significant drop in void content could be achieved by continuing the resin flow after the reinforcement has been completely wet out. In this way, the resin advects out voids and more time is allowed for dissolving any binder or excessive sizing on the fiber surface. In industrial practice this procedure has commonly been referred to as bleeding. Chen et al. [9] studied the physicochemical variables (e.g. surface tension, viscosity, and equilibrium contact angle) controlling void mobilization and concluded that voids entrapped by a resin with lower surface tension are more deformable, and consequently, easier to wash out from the fiber bundles. According to Chen's study, resin viscosity also appears to have a great influence on the momentum transfer mechanism for void mobilization. Higher viscosities are expected to increase the compressive pressure over the bubbles and help in their mobilization. However, the trade off is that when the resin viscosity is too high penetration into the interfiber channels becomes much more difficult; and molding pressures rise to almost impractical levels. Recent experiments conducted by Blackmore et al. [11] on the detachment of bubbles in slit microchannels by shearing flows confirm many of the previous observations found in the composite materials literature. One of their major conclusions was that the fluid drag force necessary to move a bubble located in contact with both surfaces inside a narrow channel must always be greater than the adhesion force that keeps it in place. The source of this adhesion force is related to the bubble liquid-vapor surface tension and the difference between the advancing and receding contact angles as,

$$
F_{a}=2 D_{c} \gamma\left(\operatorname{Cos} \theta_{r}-\operatorname{Cos} \theta_{a}\right)
$$

where $D_{c}$ is the bubble contact diameter, $\gamma$ represents the bubble liquid-vapor surface tension, $\theta_{r}$ is the receding contact angle, and $\theta_{a}$ corresponds to the advancing contact angle. From Equation (1) it can be easily inferred that, at the same flow condition, a larger bubble has a longer perimeter and hence larger adhesion force. In contrast, small bubbles have lower adhesion force and therefore become mobile in a straight microchannel at lower fluid shear rates. Analysis of the void size and shape distributions within composite parts clearly show that small and circular voids tend to concentrate in regions away from the inlet, which is definitely a consequence of their higher mobility [12,13]. Equation (1) may also serve as a theoretical explanation to the findings of Chen et al. [9] and other authors regarding the effect of surface tension on void mobilization. However, no simple generalization can be made in the case of composites liquid molding because lower surface tensions may help in void advection, but at the same time, a liquid with lower surface tension would exhibit poor wetting on inorganic preforms and therefore induce higher porosity. In order to account for the effects of both physicochemical and flow-related 
variables on void migration, the capillary number, $\mathrm{Ca}$, defined as the nondimensional ratio of the viscous forces to the capillary forces, has often been used [9]:

$$
\mathrm{Ca}=\frac{\mu V}{\gamma},
$$

where $\mu, V$, and $\gamma$ are the polymer viscosity, the macroscopic fluid front velocity, and surface tension of the reacting mixture, respectively. Recently, Patel et al. [6] introduced the idea of using a modified capillary number, $\mathrm{Ca}^{*}$, as the nondimensional parameter to relate the viscous forces and surface forces during fluid flow with the wettability characteristics of the substrates, given by the equilibrium contact angle. The expression for the modified capillary number is given by,

$$
\mathrm{Ca}^{*}=\frac{\mu V}{\gamma \operatorname{Cos} \theta}
$$

When plotting the void area fraction measured in experiments with various model fluids injected at different velocities, Patel et al. [6] found that, regardless of the type of fluid or solid wettability, all the voidage data collapsed into a single master curve having the modified capillary numbers as the independent variable. Further experimentation carried out by the same authors [7], as well as by Manhale et al. [14], indicate the existence of a critical capillary number for void formation lying in the range of $2.5-3.5 \times 10^{-3}$. Similarly, Patel et al. [7] defined a critical capillary number value for void elimination through advection that depends on the fiber geometry and is an order of magnitude higher (e.g. $1.0-2.2 \times 10^{-2}$ ) than the critical capillary number for void formation. To attain these capillary numbers, injection of liquid must be performed at high flow rates. Model experiments with parallel plate microchannels [11] also showed that for a given channel spacing $(h)$, a critical capillary number needed to be reached in order to generate a detaching shear force sufficient to mobilize a bubble of a particular contact diameter, $D_{c}$. This critical capillary number was found to decrease linearly as $D_{c} / h$ increased, reaching a plateau as the channel spacing became smaller.

A substantial decrease of the interlaminar adhesion properties with increasing levels of voidage has been long recognized in literature, and empirical correlations of exponential type have been put forward to represent the void-property relation $[13,15]$. In most cases, these analytical predictions assume a simple relationship between mechanical performance and the bulk or overall void content as the sole independent parameter. However, given that voids are likely to originate from different sources during filling, other void characteristics such as void shape, size, and spatial distribution must be taken into account in composite durability predictions, as these individual parameters will contribute to mechanical properties and failure mechanisms in different ways. For example, Lundström and Gebart [10] report the coexistence of two types of voids formed in RTM composites with unidirectional glass-fibers: slender cylinders, located preferentially inside the fiber bundles; and, large spherical voids, found in the interstices between the fiber bundles. They noted that large bubbles, in particular, aligned themselves perpendicularly to the flow direction as a result of the periodic constrictions formed by the weave pattern of the reinforcement (i.e. the reinforcement had $5 \%$ of the fibers perpendicular to the rest of the fibers). This particular example shows the strong dependence of void morphometry 
on parameters such as the reinforcement volume fraction and architecture. Howe et al. [16], on the other hand, characterized the types of voids formed in carbon/epoxy composite parts manufactured by two different molding schemes: Autoclave molding of prepregs and RTM. They found that, at similar fiber volume fractions, voids in the autoclaved laminates were mostly asymmetric and exhibited sharper edges; whereas in the RTM parts porosity was predominantly near-spherical or elliptical. By comparing the reductions in the interlaminar shear strength values (ILSS) caused by voids in each molding scheme, Howe et al. [16] concluded that voids with a more circular shape had less effect on reducing the ILSS than asymmetric voids.

In terms of the void concentration, Varna et al. [12] indicated that RTM-specimens with low void content can achieve high strength levels when tested under uniaxial tension, but also argued that the formation of large transverse cracks can make them brittle, thus leading to low strain to failure. Meanwhile, for laminates with slightly more voids, the fracture mechanism is initiated with the appearance of many small cracks connecting large spherical voids; although, some of these cracks connected small cylindrical voids only. Often, claim the authors [12], a small crack is arrested at a weft bundle, and the crack tip is somewhat displaced with respect to the first crack. This irregularity in the crack growth mechanism results in a lower stress concentration and stress level at the fiber bundles, which in turn explains the nominally higher transverse strain to failure of specimens containing slightly more voids. Very few works in the literature have addressed the topic of interlaminar shear strength reduction by discrete voids, or the influence of void shapes in the failure initiation mechanisms. Wisnom et al. [17], for example, observed that in glass/epoxy and carbon/epoxy specimens with discrete inclusions, longer voids could cause premature failure to initiate from the defect, not only by a stress concentration mechanism but also from the reduction in the load-bearing cross-sectional area.

Successive steps of packing and bleeding - among other procedures - have been recommended to composite manufacturers as a way to obtain parts with low void content [18]. Usually, after the mold is full, the resin injection is stopped and the vents are closed. Thereafter, packing starts by continuing resin injection while keeping the vents shut. After the packing is complete, the vents are released to let the resin bleed. Although effective removal of macrovoids and significant porosity reductions are attained by this method, the packing and bleeding sequence increases the total cycle time as well as the fixed costs represented in the wasted resin (ca. US 40-50 per pound). The utilization of surfactants in the flow front [9,19], surface waxing of mold walls [20], and vibration-assisted RTM [20,21] are also alternatives for void reduction that have been implemented with mixed results. An optimum condition of fluid impregnation wherein both micro- and macroflows are balanced seems imperative for minimizing void formation during a given mold filling process. As described before, experiments with different fiber architectures and model fluids favor the existence of a critical range of capillary numbers wherein small void contents can be achieved [7,9,22]. Notwithstanding the physical barriers imposed by the surface and viscous forces, there have been successful attempts to overcome void formation at high-speed molding. Among such strategies, the change of the inlet location $[23,24]$ and the utilization of higher injection pressures $[5,25]$ have demonstrated a significant reduction both in mold filling times and void inclusions. Further, other alternatives that can be readily implemented like preheating the preform to remove volatiles from the sizing system prior to resin injection [26]; and also, applying a "postfill" cure pressure or "packing pressure" after the mold fill operation, have been also shown to 
be effective in superseding fluid front influences which otherwise would trigger void formation [13,26,27].

To the best of the author's knowledge, a systematic investigation to discern the effects of different postfill pressure levels on void mobilization and porosity reduction in RTM composites have not yet been undertaken. In the current work, we study the extent of processing-induced defect reduction attained by applying a postfill cure pressure at the following four different levels: $0,300,568$, and $781 \mathrm{kPa}$. All composite parts were reinforced with commercial glass-fiber random-mats, and molded with a high-speed molding setup that allowed the filling of a $58 \mathrm{~cm}^{3}$ mold at a constant flow rate in an average of less than $10 \mathrm{~s}$. These fill times are consistent with current industrial molding cycles for small to medium sized RTM-parts. Microscopic image analysis of the average void content, size, and shape distribution at different locations along the part radius, as well as through-the-thickness, revealed interesting phenomena such as the advection of voids and a reduction in the average void size only as the result of a postfill cure pressure. A preferential concentration of remaining voids towards the mold walls-independent of the packing pressure-was noted, which is perhaps indicative of the onset of either secondary flow phenomena (e.g. microfountain flow), or unfavorable wetting conditions associated with the lower surface tension of the mold walls when covered with moldrelease agent.

\section{EXPERIMENTAL STUDIES}

\section{Procedure for Composite Fabrication}

Figure 1 depicts the major components of the high-speed molding setup used to fabricate random-mat glass/epoxy RTM composites. This setup is similar to the one described in a recent publication [13], and includes a hydraulic press and a rectangular aluminum mold containing the fiber preform. The molding press consisted of two hollow cylinders, two plungers, and a 40-ton hydraulic (ARCAN, Model CP402) press that provided the force necessary to inject the reacting mixture into a disk-shaped mold cavity. Prior to the injection, EPON 815C epoxy resin (Shell Chemicals) and EPICURE 3282 curing agent were separately loaded into two hollow stainless-steel cylinders. These cylinders had inner diameters of $55.5 \mathrm{~mm}$ for the resin and $25.5 \mathrm{~mm}$ the curing agent, such that a mixing ratio of $4.7-1$ by volume was achieved with the constant displacement rate of the plungers. During molding, a linear velocity of $2 \times 10^{-3} \mathrm{~m} / \mathrm{s}$ of the hydraulic ram was maintained through most of the $152.4 \mathrm{~mm}$ stroke, which yielded an injection rate of approximately $5.32 \mathrm{~cm}^{3} / \mathrm{s}$.

The resin and curing agent flows merged in a T-connector and thereafter passed through a Statomix ${ }^{\circledR}$ inline mixer (ConProTec, Inc.) comprising 32 alternating helical segments placed inside a $155 \mathrm{~mm}$-long polypropylene tube with $5 \mathrm{~mm}$ inner diameter and $8 \mathrm{~mm}$ outer diameter. Transient pressure profiles of the completely mixed polymer $(\mu=960 \mathrm{cP})$ were recorded with a pressure transducer located at the injection port. The center-gated diskshaped aluminum molds were made of two square walls with dimensions: $228.6 \mathrm{~mm} \times 228.6 \mathrm{~mm} \times 6.35 \mathrm{~mm}$, separated by a $3.18 \mathrm{~mm}$-thick aluminum spacer plate. To create the mold cavity, a $152.4 \mathrm{~mm}$ diameter disk was cut out of the center of the aluminum spacer plate. Both the top and bottom aluminum parts had a circular groove of $184 \mathrm{~mm}$ in diameter and $0.3 \mathrm{~mm}$ in depth carved out to accommodate a rubber seal. Inlet 


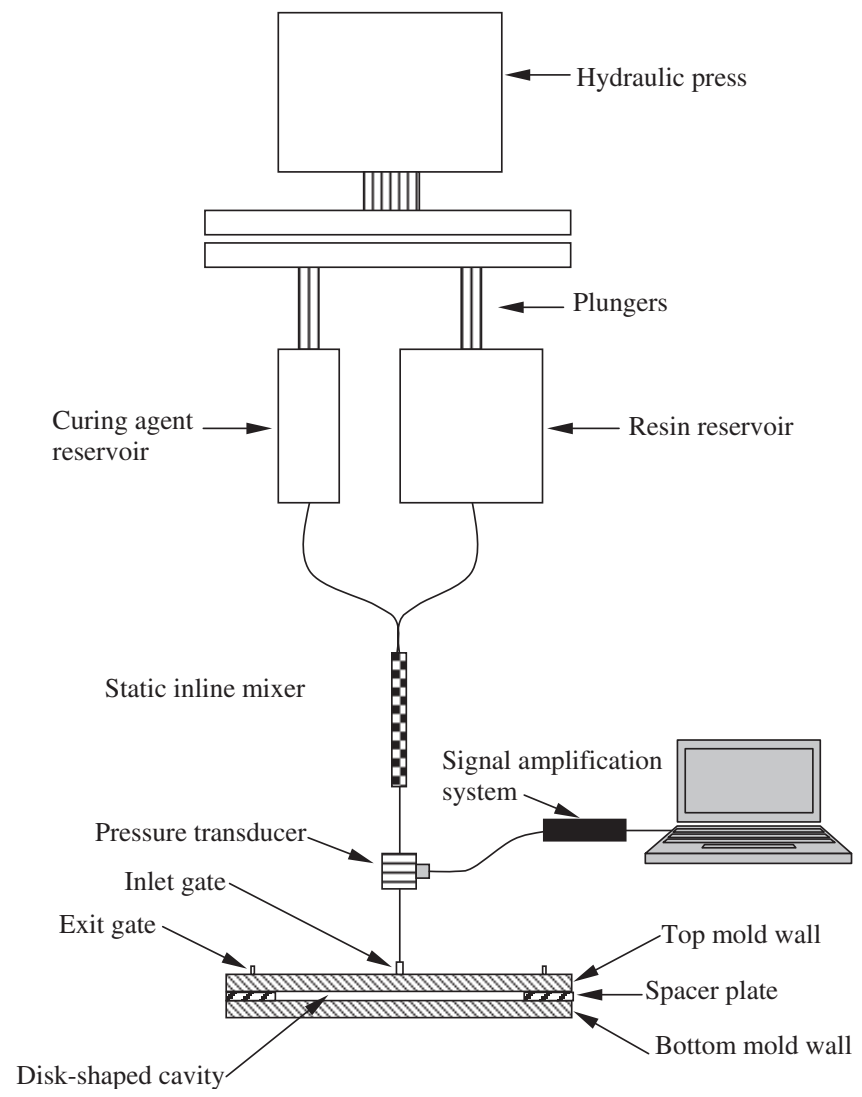

Figure 1. Experimental molding setup used to fabricate composite disks.

gate and exit vents were drilled and tapped on the top mold wall. The inlet was located in the center of the disk and had either plastic or brass barbed fittings connected to the feeding hose (ID $=6.2 \mathrm{~mm}$ ). The four symmetrical vents $(I D=1.6 \mathrm{~mm}$ ) were positioned $90^{\circ}$ apart at a radius of $88.9 \mathrm{~mm}$.

The reinforcement utilized in this study was a randomly-oriented chopped glass-fiber mat (FiberGlast part \#250), having a planar density of $0.459 \mathrm{~kg} / \mathrm{m}^{2}$. Among the attractive features of this preform are the planar isotropy and relatively low cost. Four circular layers $(152.4 \mathrm{~mm}$ diameter) of the preform were cut and stacked into the mold cavity, which represented a fiber volume fraction of approximately $18 \%$ in all composite parts fabricated. To impregnate the preform, the resin and curing agent mixture was injected into the mold cavity by a constant displacement of the plungers as described above. On average, filling the mold cavity with the preform took less than $10 \mathrm{~s}$, after which the exit gates were securely clamped and no resin bleeding was allowed to occur. Thereafter, the hydraulic press was run for a few additional seconds until the desired postfill pressure was reached at the inlet. A total of four postfill pressures, i.e. $0,300,568$, and $781 \mathrm{kPa}$, were used to investigate postfill pressure effects on overall void content as well as the shape, size, and spatial distribution of voids. In order to prevent anomalies due to imperfect sealing, expansion of the hoses, or even the deflection of the mold walls that 
could cause a significant drop in the postfill pressure, continued monitoring of pressure levels lasted for at least one minute before the inlet gate was finally clamped. Molded disks cured inside the molds at room temperature for $48 \mathrm{~h}$ until they reach their "green state", and then were taken out of the molds. Finally, to ensure complete cross-linking of the resin, the disks were left to postcure at room temperature for a minimum of one month before further cutting composite samples and polishing for void analysis.

\section{Transient and Postfill Pressure Measurements}

The relationship between volumetric flow rate and fill pressure during liquid composite molding is an important design parameter, which is in most cases described by a flow through porous media model given by Darcy's law. In the current study, the impregnating resin was injected at a constant volumetric rate. Therefore, given the circular geometry of the mold cavity, the filling pressure steadily increased as the flow front moved radially outward. To quantify the transient pressure profiles during filling, as well as the postfill pressure levels, a pressure acquisition and monitoring system was installed at the inlet port of the mold as depicted in Figure 1. The acquisition system consisted of a flash diaphragm pressure transducer (Sensotec BP357BR Model S), mounted on the hose between the mixer and the mold inlet gate; a custom built signal amplification system based on an AD620 in-amp; and an Omega Daqbook acquisition software installed on a laptop computer. During filling, the resin flow pressure at the mold inlet is converted to a voltage signal by the pressure transducer. This output signal is conditioned for the data acquisition system through the amplifying system, which amplifies the voltage, isolates the signal, and lowers the transducer output impedance. Pressure data were recorded at a frequency of $10 \mathrm{~Hz}$.

\section{Modified Capillary Number: Surface Tension Measurements and Fiber Wettability}

As was mentioned in the introduction, fluid front progression during preform impregnation is governed by macro- and microscale phenomena that are directly linked to the occurrence of voids. To date, most attempts to understand the mechanics of porosity formation at the flow front have been based on flow visualization experiments performed with model fluids over fibrous reinforcements of different architectures. These studies have demonstrated the existence of so-called critical modified capillary numbers for void formation and advection, which are assumed to be scalable to actual liquid composite molding. It is not clear from the current literature if these master curves relating void area fraction with modified capillary number are directly applicable to RTM molding operations involving reacting polymeric systems. For this reason, herein we investigated the range of modified capillary numbers reached with the mold press depicted in Figure 1, which will be referred to as high-fill velocity impregnation, and correlated them to the overall void volume fraction. An analogous comparison was established with data from previous experiments [13] obtained at lower injection flow rates (e.g. low-fill velocity) in a molding system comprising the same polymer, fiber type, and fiber volume fraction. First, the surface tension of the epoxy, curing agent, and the reacting mixture were measured in order to calculate the variability limits of the modified capillary number $\left(\mathrm{Ca}^{*}\right)$ during mold filling with respect to changes in the liquid surface energies. The method followed for 
these measurements is based on the well-known Wilhelmy equation, which expresses the force, $F$, exerted by the tested liquid over a partially immersed solid probe, as:

$$
F=W+P \gamma \operatorname{Cos} \theta-B
$$

where $W$ and $P$ are the weight and perimeter of the solid probe, respectively; and $B$ is the buoyancy force. This latter parameter is usually neglected if the solid is slightly immersed and dimensionally uniform. All measurements were carried out in a dynamic contact angle analyzer (Cahn Instruments, Inc., DCA-322), and the experimental procedure followed was very similar to that used for measuring contact angles on fibrous reinforcements [28]. First, an approximately $5 \mathrm{~mm}$-long flamed platinum rod with $254 \mu \mathrm{m}$ diameter was hung from the arm of a highly sensitive microbalance (i.e. $0.1 \mu \mathrm{g}$ accuracy). The clean platinum rod has a high surface energy, and thus, it is expected that the contact angle of both the monomers and the prepolymer against the metal surface would be zero. Once the experiment started, $3 \mathrm{~mm}$ along the platinum rod axis was immersed into the liquid at a very slow stage velocity of $12 \mu \mathrm{m} / \mathrm{s}$. Force and time data generated during each run were automatically recorded on a computer; and with aid of a software (WinDCA 1.01), the surface tension was calculated from the most stable force reading when the liquid has receded over the platinum rod surface. Measurements for the polymer required a preliminary step consisting of loading the exact volume ratio of resin and curing agent into a $60 \mathrm{~mL}$ plastic syringe, and then manually injecting the mix through a Statomix ${ }^{\circledR}$ inline mixer, similar to the one used during composites molding for further mixing. Surface tension of epoxy polymers is known to vary with the conversion grade of the reaction. Thus, the data presented here correspond to the average of measurements taken at approximately the same time interval on at least five different batches of resin/curing agent mix. Reported results for the resin and curing agent correspond to the average of individual measurements performed with 4-6 fresh samples.

Given that the injection is performed at constant volumetric flow rate, the radial superficial velocity will be an inverse function of the radial distance from the injection port. Hence,

$$
V_{\mathrm{ave}}=\frac{Q}{A}=\frac{Q}{2 \pi H r}
$$

where $Q$ is the resin flow rate, $A$ is the cross-sectional area of the resin flow at a given time; $H$ is the thickness of the mold, and $r$ is the radius at which the capillary number is calculated. As mentioned before, Patel et al. [6] proposed the idea of a modified capillary number to account for the effect of contact angle between fiber and resin. The same approach has been also used recently by others [29] to correlate microvoids formation during RTM molding. In the current work, we recurred to single-fiber experiments based on the Wilhelmy technique to measure the wettability characteristics of the commercial fiber by the resin. In this case, the liquid probe had a known surface tension and the only parameter to evaluate was the contact angle between the glass-fiber and the liquid. Instead of a single equilibrium contact angle, $\theta$, this test allows to record both the advancing $\left(\theta_{a}\right)$ and $\left(\theta_{r}\right)$ receding contact angles. The fact that the Wilhelmy method provides an estimate of a dynamic contact angle constitutes a major advantage when trying to extrapolate the results to a macroscopic scale (e.g. moving liquid fronts during 
Table 1. Physicochemical and flow parameters for low- and high-speed RTM processing of glass-fiber random-mat composites.

\begin{tabular}{|c|c|c|c|c|c|}
\hline Fluid & $\begin{array}{c}\text { Viscosity } \\
\mu(\mathrm{cP}) \\
\text { at } 25{ }^{\circ} \mathrm{C}\end{array}$ & $\begin{array}{c}\text { Surface } \\
\text { Tension } \\
\gamma(\mathrm{mN} / \mathrm{m}) \\
\text { at } 25^{\circ} \mathrm{C}\end{array}$ & $\begin{array}{l}\text { Advancing } \\
\text { Contact } \\
\text { Angle } \\
(\theta)\end{array}$ & $\begin{array}{c}\text { Range of } \\
\text { Critical } \\
\text { Capillary } \\
\text { Number } \\
\text { (Low-speed Fill) } \\
\text { [13] }\end{array}$ & $\begin{array}{c}\text { Range of } \\
\text { Critical } \\
\text { Capillary } \\
\text { Number } \\
\text { (High-speed Fill) }\end{array}$ \\
\hline$\overline{\text { EPOI }}$ & $500-700^{\dagger}$ (Avg. 600) & $0.6 \pm 0.5^{*}$ & $34^{\circ} \pm 5^{\ddagger}$ & $0.0025-0.036$ & $0.068-0.95$ \\
\hline EPICU & $\begin{array}{c}4000-4900^{\dagger} \\
(\mathrm{Ca} \text { based on } 4000 \mathrm{cP})\end{array}$ & $180+1$ & $\begin{array}{c}0^{\circ} \\
\text { (assumed) }\end{array}$ & $0.012-0.167$ & $0.317-4.4$ \\
\hline $\begin{array}{l}\text { Polymeric } \\
\text { mixture }\end{array}$ & 960 & $36.3 \pm 0.8$ & $\begin{array}{l}\sim 34^{\circ} \\
\text { (assumed) }\end{array}$ & $0.009-0.064$ & $0.12-1.7$ \\
\hline
\end{tabular}

${ }^{\dagger}$ Reported value from manufacturer, "Measured.

composite fabrication imply dynamic contact angles). Therefore, herein all calculations involving the modified capillary number include the advancing contact angles measured for the pure epoxy resin and fibers from the random-mat preform. These advancing contact angles have been already presented elsewhere [28]. It is worth noting that the fibers were relatively small in diameter (e.g. $13 \mu \mathrm{m}$ ), and hence bending of the fibers was realized as a source of errors when submerging the fibers into test liquids. In consequence, a stage velocity of $2 \mu \mathrm{m} / \mathrm{s}$ was set for all experiments to avoid disturbances in the recorded force coming from viscous friction. For this reason, contact angle measurements with the reacting mixture proved impractical, as the necessary experimentation time was much longer than the gelling time. Surface tension, viscosity, and other physicochemical parameters of the monomers and polymers used in the current study are presented in Table 1.

\section{Voids Characterization}

Porosity evaluation in composite materials by means of optical image analysis has been demonstrated to be among the most accurate methods for measuring the true void content [30]. Furthermore, the image analysis technique has the advantage of providing detailed information of other important parameters such as voids distribution, shape, and size that cannot be assessed by either physical (e.g. relative density) or chemical (e.g. acid digestion) methods. Usually, voidage measurements by optical imaging imply the random acquisition of pictures over the area of interest followed by the statistical averaging of the void areas measured in each picture. In the current work, unlike most previous work, we performed image analysis over the entire cross-sections of specimens cut from the center of completely cured disks molded at each postfill pressure. Hence, all voids that could be identified at the working magnification were included in the porosity calculations. The specimens had average length and thickness of 76.27 and $3.98 \mathrm{~mm}$, respectively, and were embedded into a quick cure acrylic resin (Allied High Tech. Products, part \# 170-10000). Once embedded, the samples were polished with a series of polishing pastes (Clover Compound) with grits sizes ranging from 180 (e.g. $80 \mu \mathrm{m}$ average particle diameter) to $1200(5 \mu \mathrm{m})$ in six successive steps. After each step, the samples were set for $20 \mathrm{~min}$ in an ultrasonic cleaner at moderate sonication $(19 \mathrm{~W})$ to completely remove 
residues of the polishing compound. The optical analysis started by dividing each $76.25 \mathrm{~mm}$ long sample into five $15.25 \mathrm{~mm}$ long regions along the radial direction. Each cross-section was then entirely scanned at $200 \times$ magnification using a MEIJI optical microscope. At this particular magnification, every frame displays approximately $0.71 \times 0.54 \mathrm{~mm}^{2}$, which needed the capture of eight frames for scanning across the sample thickness (i.e. $3.98 \mathrm{~mm}$ ) at a particular radial location. Each time a void was clearly identified the picture of the region containing the void was taken. A total of approximately 1400 frames containing voids were taken from all radial locations in all samples analyzed.

In addition to the image analysis in the radial direction, voidage distribution through the specimen thickness was also recorded. To do that, frames from the top layer (defined as the area within $1.07 \mathrm{~mm}$ from the top surface), bottom layer $(1.07 \mathrm{~mm}$ from the bottom surface), or inner layer (intermediate region) of the cross-sections were investigated. In the case of the specimen not subjected to any postfill pressure, the large number of voids present made it tedious to cover the whole specimen area. Thus, only for this particular sample we resorted to a statistical approach and instead of covering the whole cross-section of the specimen, pictures covering an area of $1.43 \mathrm{~mm} \times 1.43 \mathrm{~mm}$ were taken from the top, bottom, and intermediate zones through the specimen thickness. As described before, the voids entrapped within a particular cross-section were discriminated and their images captured using a PC-based CCD camera attached to the optical microscope. Each picture was then processed using the image analysis software Image Tool, which allowed simultaneous measurements of voids' area $(A)$ and maximum length $\left(L_{\max }\right)$. Due to the planar randomness of the preform and the shape of the mold, the flow is assumed to depend only on the radial location for each disk. Hence, the void volume fraction at each cross-section was assumed to be linearly equivalent to the ratio of the total void area by the entire area of the cross-section [31].

An equivalent diameter, $D_{\text {eq }}$, was defined in order to classify the void size distribution for each specimen. Using the measured surface area for each captured void, the equivalent diameter is calculated by the expression:

$$
D_{\mathrm{eq}}=\sqrt{\frac{4 A}{\pi}}
$$

where $A$ is the measured area of the void. Large voids are defined as those voids with an equivalent diameter 10 times greater than the fiber average diameter, i.e. $D_{\text {eq }}>50 \mu \mathrm{m}$; while voids with an equivalent diameter lower than $75 \mu \mathrm{m}$ (five times the average fiber diameter) are regarded as small voids. Intermediate equivalent diameter values, i.e. $75 \mu \mathrm{m}<D_{\text {eq }}<150 \mu \mathrm{m}$, correspond to medium size voids. In addition to the equivalent diameter, we introduced the shape ratio, $R_{s}$, as a quantitative measure of the geometrical circularity of the voids. The shape ratio is defined for each void as the equivalent diameter obtained from Equation (3) divided by the maximum measured length, $L_{\max }$ within a void,

$$
R_{s}=\frac{D_{\mathrm{eq}}}{L_{\max }}
$$

An ideal circle is represented by $R_{s}=1$. Thus, our criteria for circularity included shape ratios above $0.75\left(0.75<R_{s}<1\right)$, and all voids within this range were considered as circular voids. Usually, these circular voids are found in matrix-rich zones. If the minor axis is 
much smaller in comparison to the maximum length, the circular symmetry is lost, and the void shape turns into an ellipse. This helped define the category of elliptical voids, which corresponds to voids with shape ratios in the range: $0.5<R_{s}<0.75$. The final class of voids found in the current study is that of voids with very low shape ratios. In general, shape ratios of less than $0.5\left(0<R_{s}<0.5\right)$ are observed in highly elongated features where the longest axis is at least one order of magnitude higher than the minor axis. Those voids with low $R_{s}$ were referred to as irregular.

Figure 2 depicts images of composite cross-sections with various voidage levels, as well as voids with sizes and shapes representative of the classification presented above. In these images, the continuous phase (i.e. polymeric matrix) appears as a gray background, whereas the white circular and elliptical objects correspond to glass-fibers oriented perpendicularly and in an angle, respectively, to the cross-section. The groups of fibers observed in close proximity to one another exhibit similar orientations and correspond to fiber tows bundled together within the random-mats. A typical example of circular voids is presented in Figure 2(a). The void on the left end of the picture has a diameter of ca. $130 \mu \mathrm{m}$, which is an intermediate size according to our classification. The other void in the same picture corresponds to a small void with a diameter no greater than four times the fiber diameter (ca. $60 \mu \mathrm{m}$ ). Note that these two voids are both located within the matrix area. Voids that are trapped in the intertow spacing are forced to conform to the channel geometry. As it is seen in Figure 2(b) they are mostly small and elliptical. In Figure 2(c) a couple of small and irregular voids are depicted. The irregular voids were, in most cases, found either inside the intratow areas or engulfing a group of fibers from a tow. It appears as if they were originated from rounded bubbles that are pushed into the tows by a high shear force during the resin flow. Figure 2(d) exemplifies a high voidage area taken from the composite sample molded without applying a postfill pressure.

In the current study, all microscopic void analysis is performed on images taken through-the-thickness plane of the molded disks. On through-the-thickness plane, intratow voids appear as smaller, circular, or irregularly shaped voids inside fiber bundles. Yet, these voids form at high-speed filling conditions, and extend longitudinally along the fibers [9]. In order to depict the morphology of such voids, planar views of the microstructure is obtained as shown in Figure 2(e) and (f). The voids, as expected, are cylindrical in shape as shown in Figure 2(e), with their major axis running parallel to the microchannel spacing formed by the fibers. It is very interesting to note that two clear spherical caps are formed in both ends of the void, resembling the shapes of the advancing and receding meniscus formed during the void displacement. In fact, existence of elongated, cylindrical intratow voids indicates the high-speed nature of the impregnation process. Finally, Figure 2(f) suggests that most intratow voids appeared to be eliminated from the intratow space after the postfill pressure was applied.

\section{RESULTS AND DISCUSSION}

\section{Transient Inlet Pressure}

Positive displacement of the plungers by the molding press ensured that infusion of the reacting polymer through the random-fiber preform would take place at a constant volumetric flow rate. Due to the circular mold geometry, the resin front advances through the dry preform and towards the exits; and in most cases, a radial liquid front symmetry 
(a)

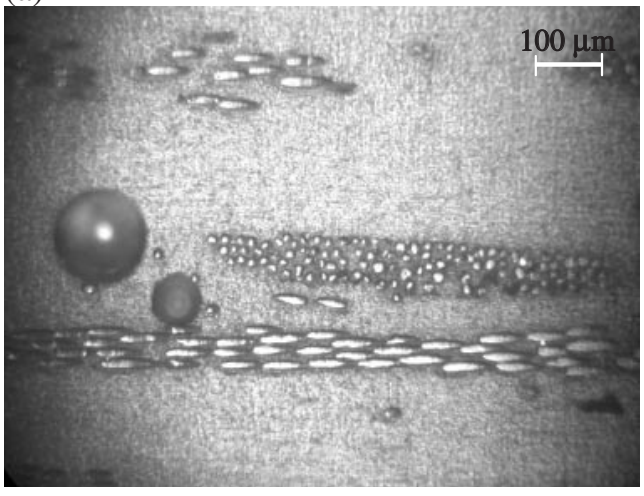

(c)

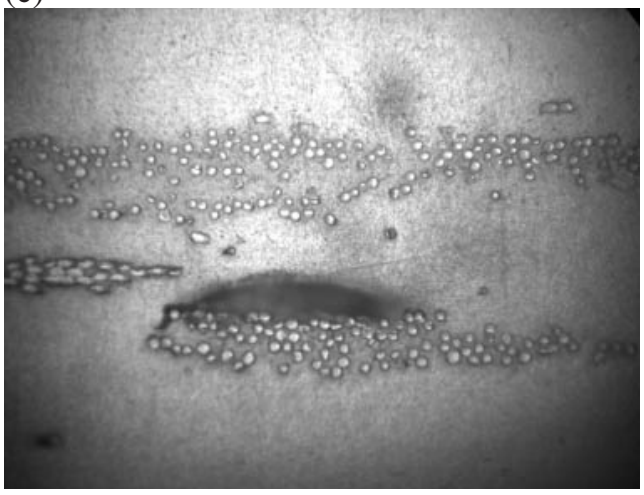

(e)

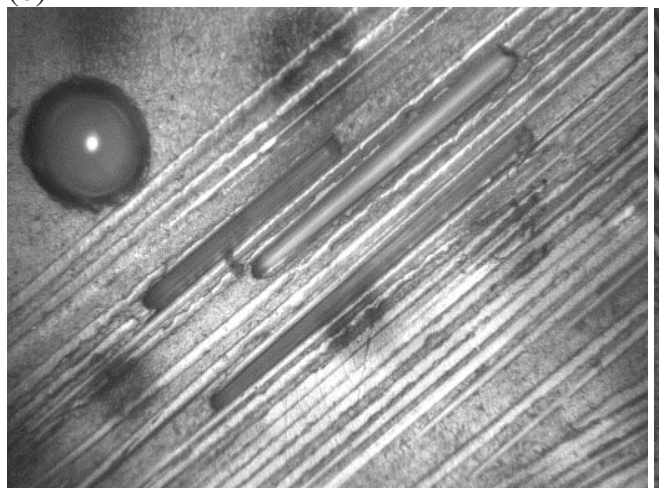

(b)

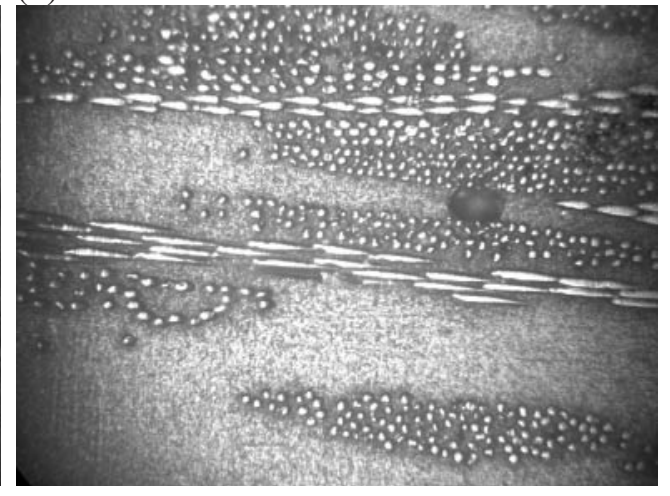

(d)

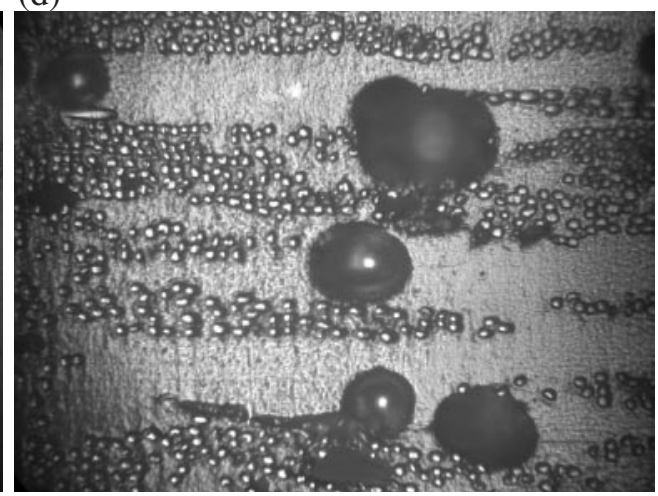

(f)

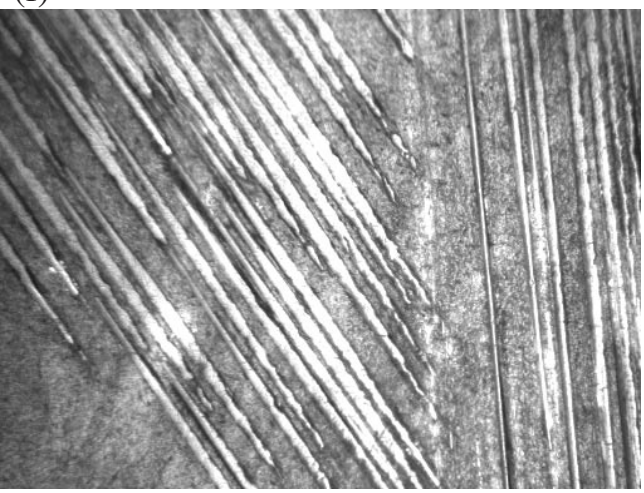

Figure 2. Representative micrographs from different postfill pressure specimens at $200 \times$ magnification. Figures (a)-(d) are taken from through-the-thickness images and Figures (e)-(f) are taken from planar images. (a) Circular voids of different shapes located in matrix-rich area; (b) Ellipsoidal void in the intertow space; (c) Irregular void engulfing a tow of fibers; (d) Example of high voidage area in composites molded without a postfill pressure; (e) Intratow voids in composites without postfill pressure; (f) Intratow view of composite sample with highest packing pressure. 
can be assumed. Three transient positions of the advancing flow front inside the mold are depicted in the top part of Figure 3. Very close to the entrance, the impregnating front moves at high velocities yielding much faster local velocities at the mold cavity midplane. Further away from the injection port, the advancing front velocity decreases monotonically at radial positions (e.g. bottom part of Figure 3). The total energy of the liquid is reduced due to the mold geometry, together with the resistance exerted by the dry preform surface. This energy dissipation becomes greater as the dry surface area opposing the flow increases radially, until the fluid impregnates the whole solid and finally reaches the vents.
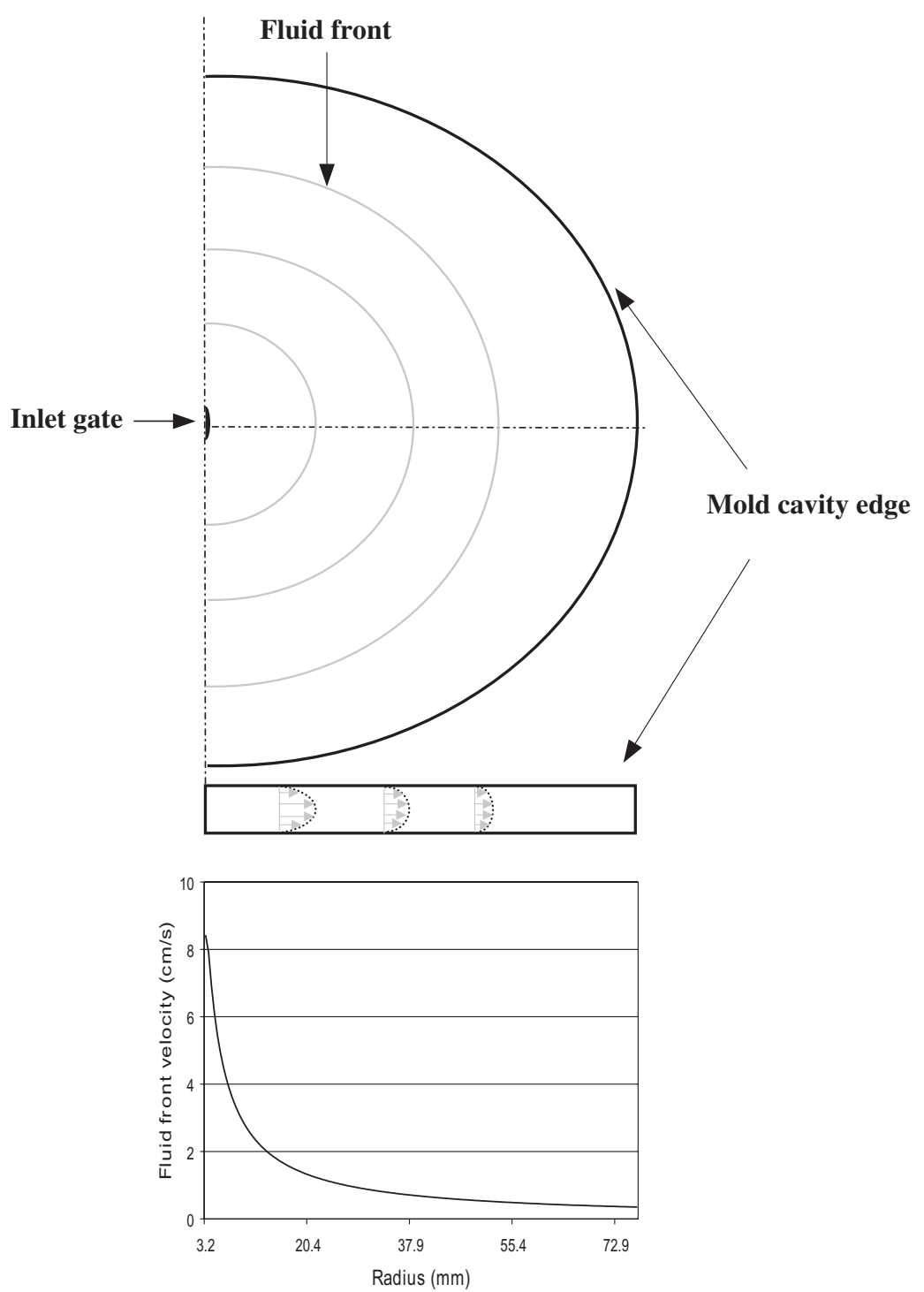

Figure 3. Advancing fluid front superficial velocity at different radial positions inside the mold. 
In order to maintain a constant impregnation rate, the pressure applied to the liquid must also increase so sufficient energy is provided to impregnate the solid. Transient inlet pressure readings of four different mold-filling experiments are shown in Figure 4(a). In this figure, four different zones that are common to all filling experiments can be identified, inter alia, the injection or fill zone, the peak pressure zone, the plateau region, and the step zone. Within the fill zone, pressure increases at a very steep rate. Note, however, that the change in injection pressure with respect to time was the same for all molded composites. Thus, considering that these parts had the same fiber volume fraction, it can be concluded that, independent of random variations in permeability, the flow field was approximately the same for all filling operations. This latter result is significant because it precludes the probability of obtaining different voidage levels in molded parts due to uncontrolled flow behavior during filling. After the fill zone, pressure readings start showing some discrepancies between different experimental runs. Note that although each molding experiment took approximately the same time to reach the peak pressure (e.g. 18s), the peak value was not the same among experiments. In Figure 4(b), a close-up of the mold vent region illustrates the relative dimensions of the narrow opening and the mold cavity. Narrower flow channels formed between the spacer plate and the top mold wall impose greater resistance to flow that translates into higher-pressure drops. Thus, peak values recorded in Figure 4(a) are more suggestive of

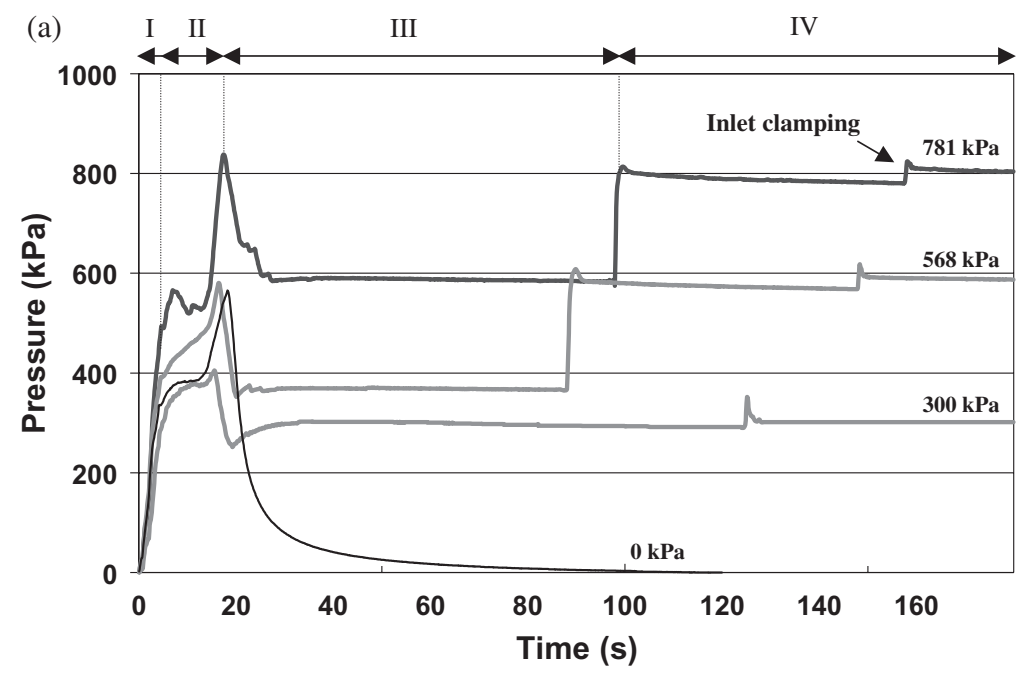

(b)

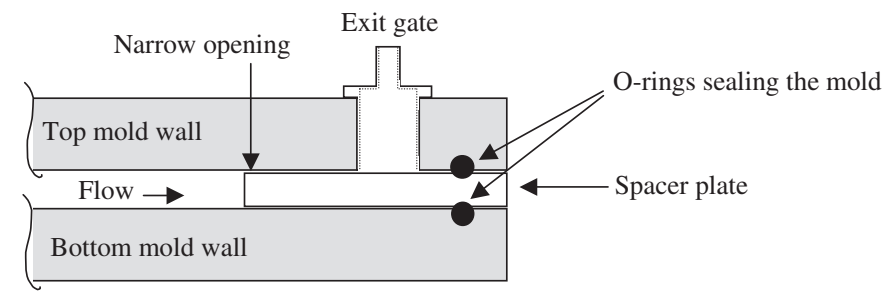

Figure 4. (a) Inlet pressure profiles during molding; (b) Close-up of air vent region. 
random variations in the constricted channel spacing than to any direct relationship with the process variables. It can be inferred from Figure 4(b) that channel spacing will depend on the deformability of the rubber o-ring utilized to seal the mold. The applied force and the condition of the o-ring during mold assembly are believed to affect the thickness of this narrow channel, and thus the peak pressure reached in zone II.

Once the resin starts flowing through the narrow channel and out to the vents, the molding press is turned off, stopping resin injection. This event marks the onset of zone III. As seen in Figure 4(b), between the time injection ceases, and the moment all vents are securely closed, there is a monotonic decrease in the recorded pressure that is consistent with the tendency of the system to attain a balance between inlet and outlet pressures. Thereafter, the pressure leveled-off to a plateau pressure value, and remained constant until the commencement of the step zone (i.e. zone IV). In the case of experiments with zero postfill pressure no compressing action took place, and as a consequence, the pressure continued decaying asymptotically.

Zone IV began when the resin inside the mold was compressed to the desired postfill pressure level (e.g. step zone). Two or three minutes after the postfill pressure is applied the injection port was clamped. The sudden contraction of the mold opening is registered in the pressure charts (Figure 4(a)) as a minute pressure peak (ca. $60 \mathrm{kPa}$ ) towards the end of the experiment. In summary, the transient pressure has shown that both the mold filling, as well as the establishment of a final postfill pressure, were adequately controlled with the current molding setup and molding procedures.

\section{Molding Speed, Capillary Number, and Overall Void Content}

One of the advantages of RTM over similar composites fabrication techniques lies in the possibility of attaining short cycle times. Therefore, a critical step during RTM molding is to impregnate the preform as quickly as possible while minimizing undesirable features such as a race-tracking, high void content or incomplete wet-out that may result in premature failure or poor overall performance [32,33]. In an earlier study [13], we investigated the effect of volume flow rate and postfill cure pressure on the mechanical properties and overall void content of RTM composites with identical mold geometry, fiber architecture, volume fraction, and epoxy system, as the parts molded in the current work. Therein, four different constant injection rates of $0.067,0.2,0.6$, and $1.0 \mathrm{~cm}^{3} / \mathrm{s}$ representing more than an order of magnitude change in fill times were used. Corresponding fill times for these injection rates were: 900, 300, 100, and $60 \mathrm{~s}$, respectively. Tensile strength and stiffness reductions in the order of 14 and $13 \%$ due to increased fill rates suggested that an injection rate increase had detrimental effects possibly associated to increased void formation. Conversely, significant improvements in the order of $13-15 \%$ were observed for the strength and elastic modulus when a range of postfill cure pressures (e.g. $228-683 \mathrm{kPa}$ ) was applied. With the objective of evaluating to what extent the application of a postfill cure pressure can be regarded as an efficient method to reduce the flow-induced porosity in composite materials, data from molding experiments carried out at low-speed (e.g. $0.20 \mathrm{~cm}^{3} / \mathrm{s}$ ) in previous work [13] are compared to analogous RTM experiments conducted at injection rates almost thirty times higher (e.g. $5.32 \mathrm{~cm}^{3} / \mathrm{s}$ ). There is approximately a 30-fold reduction in the fill time between the low- and high-speed molding schemes, which we consider to be significant in terms of extrapolating these results to the industrial fabrication scale. 


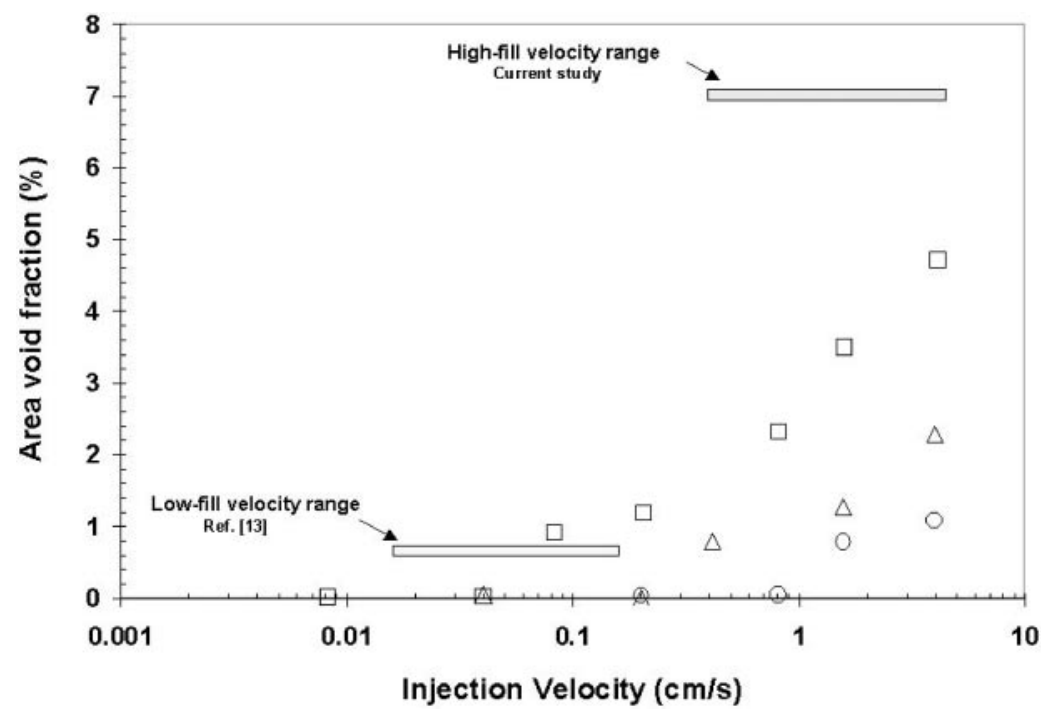

Figure 5. Effect of injection velocity effect on porosity formation. Data for model fluids taken from Rohatgi et al. [34]: $\square$ Silicone oil; $\mathbf{D}$ DOP oil; $\bigcirc$ Ethylene glycol.

With the exception of the work by Rohatgi et al. [34], not many of the flow front visualization studies presented in the literature have dealt with void formation when the impregnation velocity is high. Figure 5 shows data taken from Rohatgi's work corresponding to the trend in area void fraction as a function of liquid injection velocity for silicone oil, diphenyl-octyl-phtalate (DOP), and ethylene glycol when flowing axially over a unidirectional stitched fiberglass mat (CoFab A108). In the same figure, we have incorporated the range of liquid front velocities inside the molds (e.g. maximum velocity at the entrance, and minimum close to the exit ports) during the high-speed and low-speed processing of random-fiber/epoxy composites (e.g. abscissa); and in the ordinate, we show the overall void content for those parts molded without extra packing (postfill pressure $=0 \mathrm{kPa}$ ). As suspected, the void content in the composite part strongly depends on the liquid front velocity. At low-speed fill the porosity level is below $1 \%$, which in the composites industry is regarded as a quality threshold value for parts with good mechanical performance [18]. As long as the injection velocity increases, the entrapment of air is more likely to occur and thus the void content inside the part reaches an undesirable level of $7.2 \%$, as observed in the current study.

It would be easy to conclude that reducing the injection velocity further below the low-speed velocity range used herein could completely eliminate the driving forces for void formation. Such argument has already been proven wrong by studies on air entrapment during the impregnation of fibrous reinforcement with model fluids flowing at very low velocities. In this case, the main front velocity lags behind the faster capillary flow occurring inside the narrow interfiber spacing, and extensive formation of macrovoids takes place [7]. The results presented by Rohatgi et al., shown in Figure 5 as data-points, reveal another interesting trend. The area void fraction was - as expected - an increasing function of the injection velocity for all test liquids utilized. However, the major factor determining the ultimate level of air entrapment present was the fluid's physicochemical characteristics. For instance, viscous fluids of relatively low surface tension are more 
prone to engulf air when the flow front advances at a high speed. When silicone oil (e.g. viscosity $=193.4 \mathrm{cP}, \gamma=21.0 \mathrm{mN} / \mathrm{m})$ is injected at a velocity of $4 \mathrm{~cm} / \mathrm{s}$ the void area of air trapped is close to $5 \%$, whereas at the same velocity, only a $1 \%$ area void fraction was trapped by ethylene glycol (e.g. viscosity $=19.8 \mathrm{cP}, \gamma=48.4 \mathrm{mN} / \mathrm{m}$ ). These results corroborate our data, in the sense that they explain why the effect of molding speed on the final void content is so dramatic when using the commercial epoxy polymer. As presented earlier, the viscosity of the polymeric mixture used in the current study is in the order of $960 \mathrm{cP}$, which is five times greater than the model silicone fluid used by Rohatgi et al. Therefore, it seems reasonable to assume that with a higher viscosity fluid any increase in the front velocity would lead to more extensive entrapping of air than with the silicone oil. In fact, taking an average value for the injection velocity at the two molding schemes (e.g. $0.088 \mathrm{~cm} / \mathrm{s}$ for the low-speed range and $2.5 \mathrm{~cm} / \mathrm{s}$ for the high-speed range), and comparing them to similar injection velocities for the silicone oil; it is clear that while for the model fluid there is a fivefold increase in area void fraction between the low and high injection velocities, a 10-fold change in porosity takes place with the commercial resin. Other factors, such as the difference in fiber volume fraction (e.g. $20 \%$ in the current study vs. $43 \%$ in Rohatgi et al.) and the fiber orientation (e.g. random-mat vs. unidirectional) must also be considered in order to explain the higher void contents in our samples.

Using the physicochemical data listed in Table 1 for the polymer, and the modified capillary number $\mathrm{Ca}^{*}$ given in Equation (5), we calculated the variability limits of the nondimensional capillary number for both the high- and low-speed impregnation flows. The upper and lower limits of the modified capillary number correspond to the values measured at the injection port and close to the exit, respectively; and are represented in Figure 6 as a gray band. The data points in the same plot depict experimental results obtained by Rohatgi et al. [34] for the void area fraction as a function of the modified capillary number in moldings carried out with the same unidirectional glass-fiber reinforcement and model fluids mentioned above. These authors make a distinction between the types of voids formed at different capillary numbers: empty symbols correspond to porosity levels coming from macrovoids, which are mostly circular and highly mobile. Whereas the filled symbols, depict the area fraction of microvoids formed when the leading flow front takes place in the larger gap between tows (e.g. viscous force greater than capillary force).

From Rohatgi's data it can be inferred that reinforcement impregnation at extremely low capillary numbers favor extensive voidage accumulation in the parts, primarily composed of circular macrovoids. As the impregnation velocity increases, so does the capillary number, and the macrovoids start to mobilize and porosity values reduce considerably. Critical capillary numbers for void mobilization are in the order of $1.0-2.2 \times 10^{-3}$, as observed in this plot, and also corroborated by other studies $[7,14]$ mentioned in the introduction. Next to the critical capillary number of void mobilization there is a zone for "optimum" impregnation wherein the void content fluctuates around $1 \%$. According to theoretical calculations [29] relating capillary number to the time ratio describing the competition between intratow and intertow flow fronts $\left(\Delta t_{l T, \mathrm{~T}} / \Delta t_{l T, \mathrm{C}}\right)$, the range of low void content is located in a region of capillary numbers between $6 \times 10^{-3}$ upto 0.03 . Further increase in the injection velocities leads to higher capillary numbers, which triggers the entrapment of air, and hence the void area fraction starts augmenting. The types of voids formed at these high capillary numbers are quite distinct, and mostly correspond to ellipsoidal microvoids [34]. 


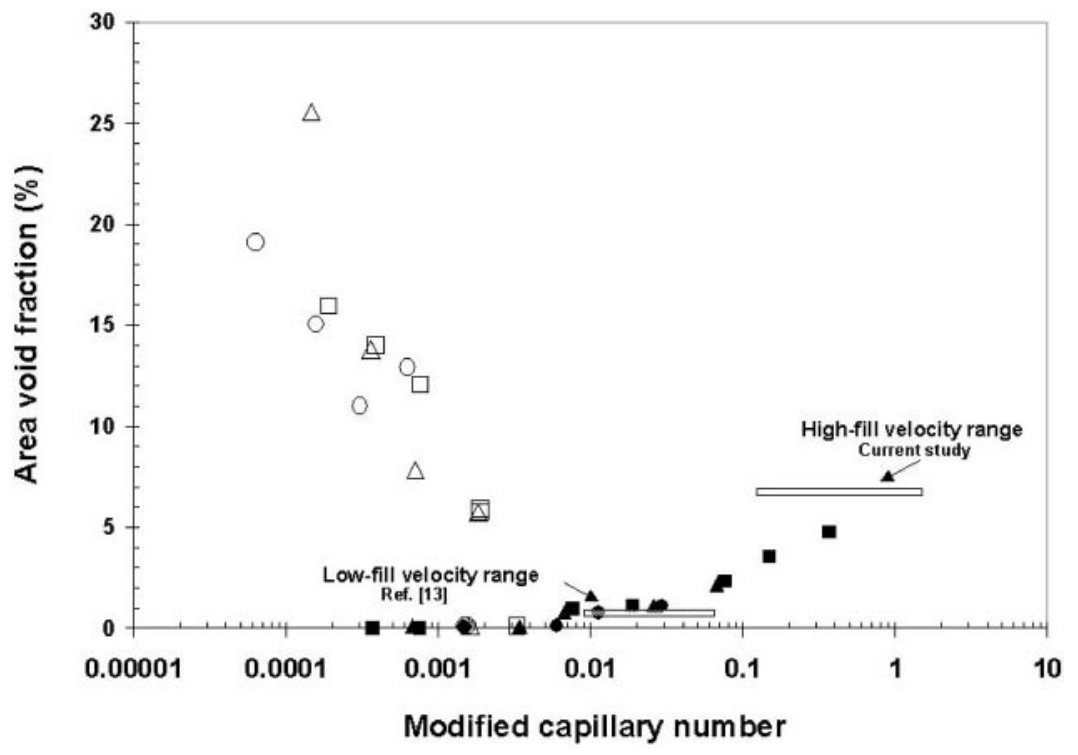

Figure 6. Effect of capillary number on porosity formation. Data for model fluids taken from Rohatgi et al. [34]: Silicone oil, macrovoids; $\mathbf{\square}$ Silicone oil, microvoids; $\triangle$ DOP oil, macrovoids; $\triangle$ DOP oil, microvoids; Ethylene glycol, macrovoids; - Ethylene glycol, microvoids.

Comparing the void area fraction formed at the range of capillary numbers established during the molding process at high speed, with the air entrapment levels occurring within the low-speed velocity range, the main conclusion that can be drawn is that our data matches reasonably well with the trends observed by other authors [9,34]. That is, at capillary numbers well above the so-called "optimum" range the void content is very high (e.g. more than 7\% for the high-speed velocity range). Whereas, for the low-speed molding, the capillary numbers were all within, or very close to, the "optimum", and therefore, the void content was almost negligible (e.g. around $0.7 \%$ ). Note that the results described so far are only for the overall void content of parts molded at either high- or low-speed velocities, but not subjected to a postfill pressure. These void contents obtained in composites molded without any further packing pressure will later be used as the baseline to compare the extent of porosity reduction achieved when a postfill pressure is applied. Table 2 lists the average voidage values for composites molded at either high or low speed and the corresponding postfill pressure levels applied. The main trend inferred from these results is that, independent of the injection mode or initial void content value, the methodology of applying a postfill pressure significantly reduces the final voidage levels in the composite parts. For instance, applying a packing pressure of $455 \mathrm{kPa}$ causes a reduction of almost $60 \%$ of the initial void fraction in parts molded at low-speed. Similarly, reductions in the order of $73-77 \%$ were achieved when the postfill pressure applied to composites molded at high-speed was in the range of $300-800 \mathrm{kPa}$. There is some evidence in the literature $[18,26,27,35]$ suggesting that high molding pressures $(0.1-0.9 \mathrm{MPa})$ may reduce porosity in composites by a mechanism involving the disruption of void nucleation and growth dynamics, as well as by the increase in the driving force for mass transfer taking place when the pressure outside the voids is higher than that of the air in their interior [3]. Lundström and Gebart [10] talk about a compression effect 
Table 2. Void volume fraction of low- and high-speed RTM composites with different postfill pressures.

\begin{tabular}{|c|c|c|c|c|c|}
\hline \multicolumn{3}{|c|}{ Low-Speed Fill Composites $^{a}$} & \multicolumn{3}{|c|}{ High-Speed Fill Composites } \\
\hline $\begin{array}{l}\text { Postfill } \\
\text { Pressure } \\
\text { (kPa) }\end{array}$ & $\begin{array}{l}\text { Void } \\
\text { Fraction } \\
(\%)\end{array}$ & $\begin{array}{c}\text { Percent } \\
\text { Reduction } \\
\text { from } 0 \mathrm{kPa} \\
(\%)\end{array}$ & $\begin{array}{l}\text { Postfill pressure } \\
(\mathbf{k P a})\end{array}$ & $\begin{array}{c}\text { Void Fraction } \\
\text { (\%) }\end{array}$ & $\begin{array}{c}\text { Percent } \\
\text { Reduction } \\
\text { from } 0 \mathrm{kPa} \\
(\%)\end{array}$ \\
\hline 0 & 0.7 & & 0 & 7.2 & \\
\hline 228 & 0.52 & 30 & 300 & 1.95 & 73 \\
\hline 455 & 0.32 & 57 & 568 & 2.42 & 66.3 \\
\hline 683 & 0.34 & 54 & 781 & 1.7 & 76.4 \\
\hline
\end{tabular}

${ }^{\mathrm{a}}$ Data from [13].

over the voids inside a laminate when the cure pressure is increased after filling. These authors even suggest that such increase in the final pressure could have a similar effect as applying vacuum. Some of the mechanisms described above are perhaps contributing to the final void content reduction observed within our samples. However, in order to seek a thorough explanation as to how these changes in porosity operate we have recurred to an extensive analysis of the size, shape, and radial - as well as through-the-thickness distribution of voids; and will relate these results with respect to the level of applied postfill pressure.

\section{Effect of Postfill Pressure on Void Morphometry and Radial Distribution}

Figure 7 depicts two of the basic parameters used in the current study for the void morphometry analysis as a function of the applied postfill pressure: $\Phi_{m}$ and $\Phi_{s}$. The first parameter, $\Phi_{m}$, represents the relative "shape" contribution of a particular type of void within the composite part in comparison to the other two shape categories defined. For instance, $\Phi_{m}(\mathrm{C} / \mathrm{I})$, is mathematically equivalent to the ratio of the observed frequency of circular voids to the observed frequency of voids with irregular shape. Similarly, we have defined $\Phi_{s}$ as the relative presence of voids with a particular size, with $\Phi_{s}(\mathrm{~S} / \mathrm{L})$ being equal to the observed frequency ratio of small to large voids.

Given the stochastic nature of the void formation at the flow front, these relative measurements yield a better description of the observed trends, and provide more distinctive elements to judge the extent of the beneficial effect of the postfill pressure. Focusing on the void morphology shown in Figure 7, there is a remarkable difference among the types of voids remaining in the composites after molding with or without a postfill pressure. In those parts with higher porosity (e.g. $0 \mathrm{kPa}$ postfill pressure), the amount of circular voids considerably exceeds the number of irregular voids. This indicates that the porosity formed during high-speed molding correspond to spherical voids located preferentially at matrix-rich regions. A higher occurrence of ellipsoidal voids in the same parts molded without packing explains the much lower ratio of $\Phi_{m}(\mathrm{C} / \mathrm{E})$. With the application of postfill pressure important reductions in the relative presence of all types of voids takes place, which is in accordance with the overall void content reductions listed in Table 2. There are much fewer, ellipsoidal, and irregular voids in the specimens molded with an extra packing pressure than in the baseline composites. In addition, the 


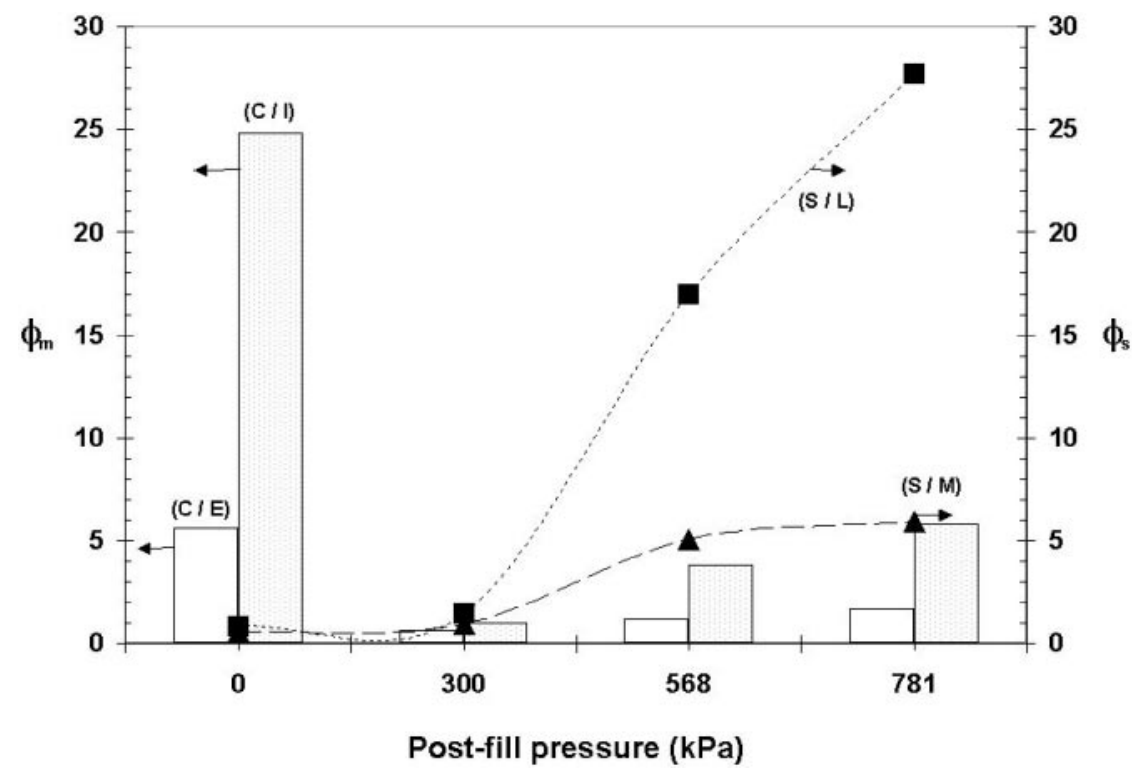

Figure 7. Relative voids shape $\left(\phi_{m}\right)$ and size $\left(\phi_{s}\right)$ contributions as a function of postfill pressure.

void morphology also changes. Irregular and ellipsoidal void content become smaller, which explains the monotonic increase of $\Phi_{m}(\mathrm{C} / \mathrm{I})$ and $\Phi_{m}(\mathrm{C} / \mathrm{E})$ with higher postfill pressure levels. With regard to the overall void size, at zero or low extra-packing (e.g. $300 \mathrm{kPa}$ ) pressure, the relative contribution of large and medium voids to the overall porosity is much more significant than when higher postfill pressures are applied. These large- and medium-size voids are represented almost completely by round voids, as was inferred from the morphology analysis, which will disintegrate into voids with smaller diameter as the surrounding pressure (e.g. postfill pressure) increases. Therefore, a sizereduction effect (e.g. void collapse) due to mass transport [10,35], between the air inside the voids and the resin, is perhaps the most plausible explanation for the steep increase observed in the parameter $\Phi_{s}$, and particularly in $\Phi_{s}(\mathrm{~S} / \mathrm{L})$.

Another mechanism responsible for porosity reduction often mentioned in the literature is the void mobilization, or advection, due to momentum transport from the resin flow into the voids in the form of shearing force. Figure 8(a) and (b) show results for the assessment of voids size and shape characteristics along the radius in RTM parts molded without a packing pressure (e.g. baseline composites). Again, the parameters $\Phi_{m}$ and $\Phi_{s}$ represent the relative presence of a specific void type in terms of shape and size, respectively. It can be observed in Figure 8(a) that at distances very close to the mold entrance the number of irregular voids is negligible, and consequently the ratio $\Phi_{m}(\mathrm{C} / \mathrm{I})$ becomes extremely large. Although the maximum resin velocity occurs in the vicinity of the injection port region, the relatively lower presence of irregular voids can be traced back to an enhanced wetting effect resulting from higher local pressures and longer contact time available between the resin and the fibers. Thus, the irregular voids tend to concentrate towards the center of the specimen and are found less frequently at positions near the exit. The ellipsoidal voids are present in uniform proportions with respect to the circular voids along the radial position (e.g. $\left.\Phi_{m}(\mathrm{C} / \mathrm{I})=7.5\right)$. 
(a)

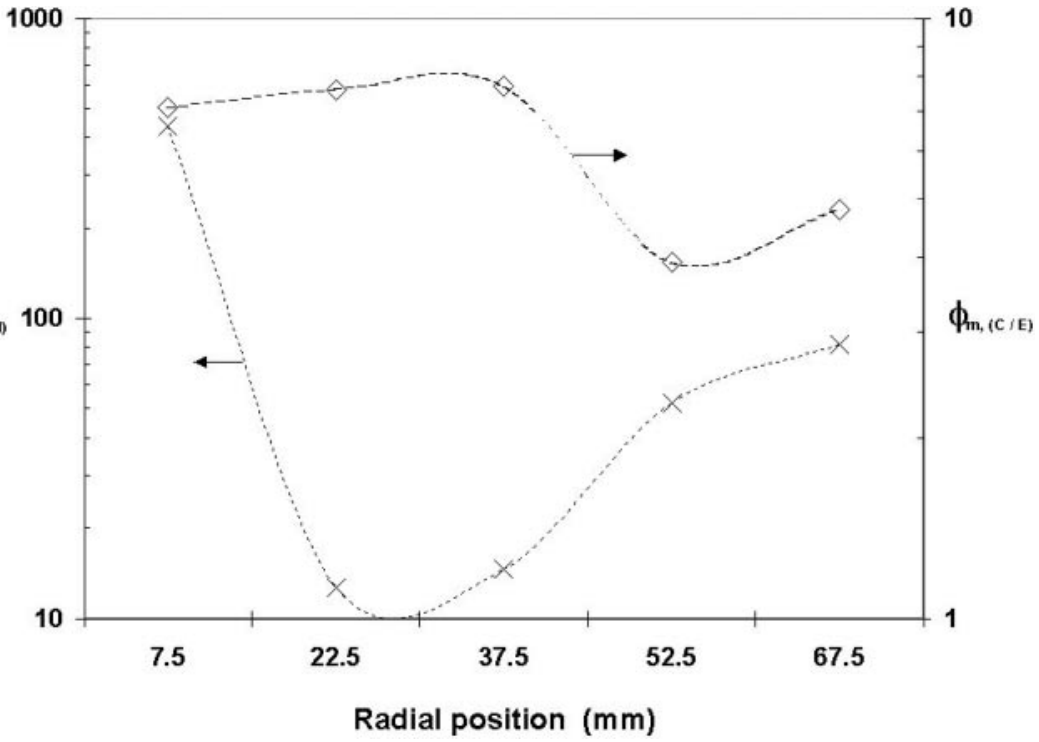

(b)

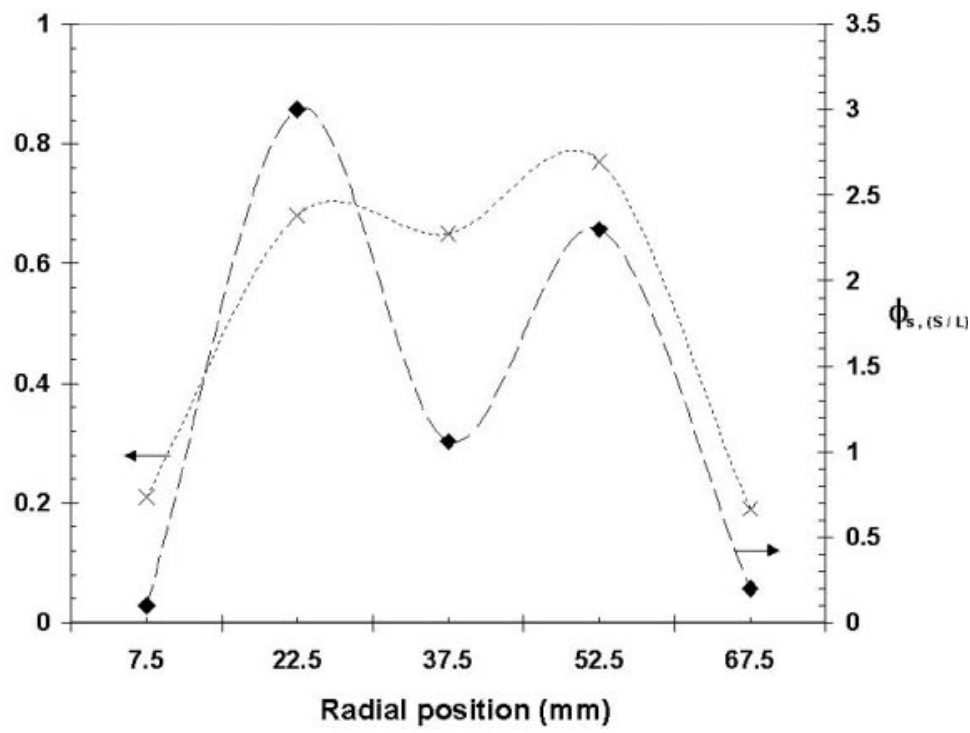

Figure 8. Relative voids shape and size contributions as a function of radial locations for composites molded without postfill pressure. (a) Shape contribution; (b) Size contribution.

Concerning the changes in void sizes along the radial locations, there is a combination of two different phenomena that dictates the particular characteristics of the results shown in Figure 8(b). On one hand, there is a selective accumulation of small voids towards the center of the specimen, coupled with a substantial drop in the presence of large voids within the same area. The large voids tend to preferentially locate at the entrance and 
towards the exit port. Hence, the values for $\Phi_{s}(\mathrm{~S} / \mathrm{L})$ are between 5 and 15 times larger in the middle part of the composite part than towards the edges. On the other hand, the same concentration of small voids in the center of the specimen translates into higher values of $\Phi_{s}(\mathrm{~S} / \mathrm{L})$; notwithstanding, the medium voids are at least three times more abundant than the large ones $\left(\Phi_{s}(\mathrm{~S} / \mathrm{M}) \ll \Phi_{s}(\mathrm{~S} / \mathrm{L})\right)$. A general observation on the spatial distribution of voids within the baseline composites (e.g. $0 \mathrm{kPa}$ packing pressure) would be that the high-velocity resin flow seems to push the more mobile small voids towards the center of the specimen; creating a zone of high voidage with predominance of irregular voids.

In contrast, the radial distribution of void and shape parameters, $\Phi_{m}$ and $\Phi_{s}$, in composites molded with various postfill pressures exhibit completely different dynamics as depicted in Figures 9(a), (b) and 10(a), (b). First of all, both $\Phi_{m}(\mathrm{C} / \mathrm{E})$ and $\Phi_{m}(\mathrm{C} / \mathrm{I})$ show linear dependences with respect to the radial position for all packing pressures (Figure 9(a) and (b)). This behavior results from a combined effect of a substantial increase in the relative content of circular voids further away from the injection port, and at the same time, the slight drop on the amount of both ellipsoidal and irregular voids towards the exit location. As the postfill pressure increases the relative contribution of circular voids become predominant, with much less amounts of ellipsoidal (Figure 9(a)) and even lesser irregular voids (Figure 9(b)) present along the radial position. The variation of void sizes $\left(\Phi_{s}\right)$ with respect to radial positions was not as definite as the one observed for the voids morphology. Figure 10(a), for instance, shows that the relative abundance of small voids is exactly the same as the medium voids all along the composite radius for a postfill pressure of $300 \mathrm{kPa}$. By augmenting the packing pressure the medium voids start to disappear, and therefore, the contribution of small voids is more evident regardless of the radial location. A comparable trend is observed in Figure 10(b) for $\Phi_{s}(\mathrm{~S} / \mathrm{L})$. At a packing level of $300 \mathrm{kPa}$ the number of small and large voids coexist in a ratio of approximately 2:1. For further increases in applied postfill pressure, larger voids are prone to collapse, and consequently the size ratio of small-to-large voids also increases up to near 25:1.

In summary, it can be said, first, that the overall void content as well as the voids shape and size distribution along the radius of the composites appreciably change with increasing levels of packing pressure. There is clearly a segregation of circular voids towards the peripheral locations in those composites where a packing pressure was applied. Whereas for those parts molded without forcing more resin into the mold after the fill (e.g. $0 \mathrm{kPa}$ postfill pressure), porosity tends to concentrate in the center of the specimen instead. This particular behavior is perhaps originated in the fact that momentum is transferred more effectively into the more mobile circular voids. Second, the relative contribution of both large and medium voids to the overall porosity value within the composite parts is greatly reduced with increases in the packing pressure; with the most significant improvements taking place for the 568 and $781 \mathrm{kPa}$ pressure levels. Such reductions in void size may be attributable to a void collapse mechanism originated by enhanced dissolution of the air inside the voids into the resin occurring at higher postfill pressures. The practical implications of these results are twofold: substantially lower overall void contents can be achieved by applying a postfill pressure, even when molding with high impregnation velocities. And also, for an equivalent overall porosity value, those parts molded with higher packing pressures will statistically contain less irregular voids as well as a smaller amount of large-sized voidage. It appears that increased postfill pressures improve wetting of fibers by forcing resin into the fiber bundles that are not fully wetted during filling. 


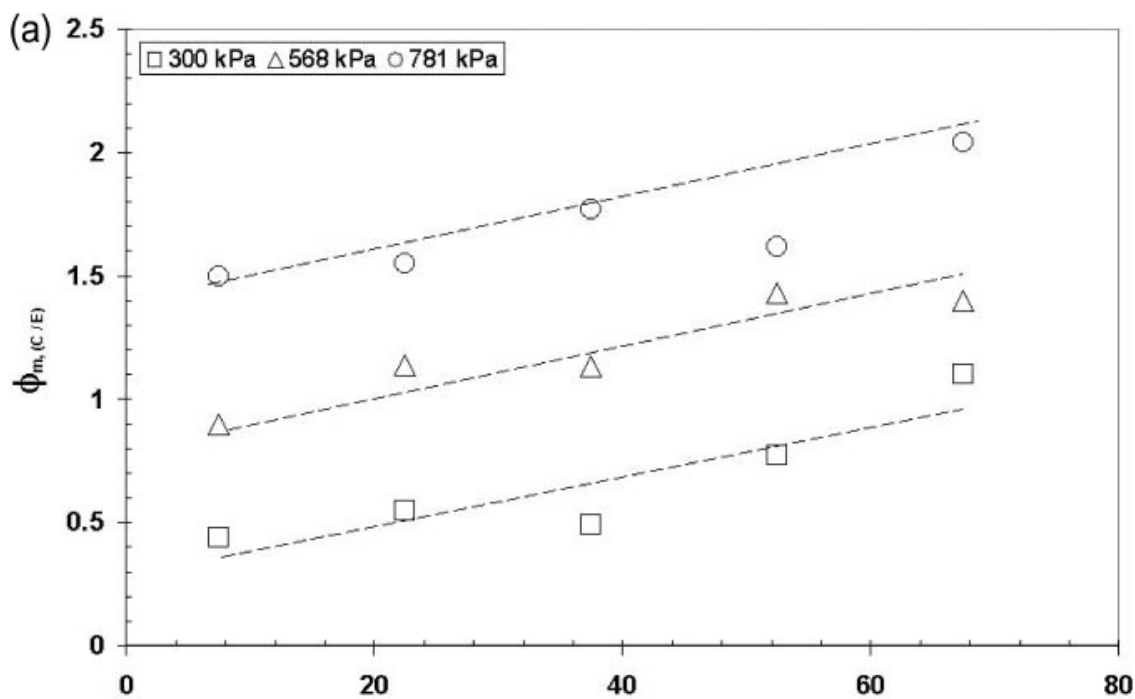

Radial position ( $\mathrm{mm})$

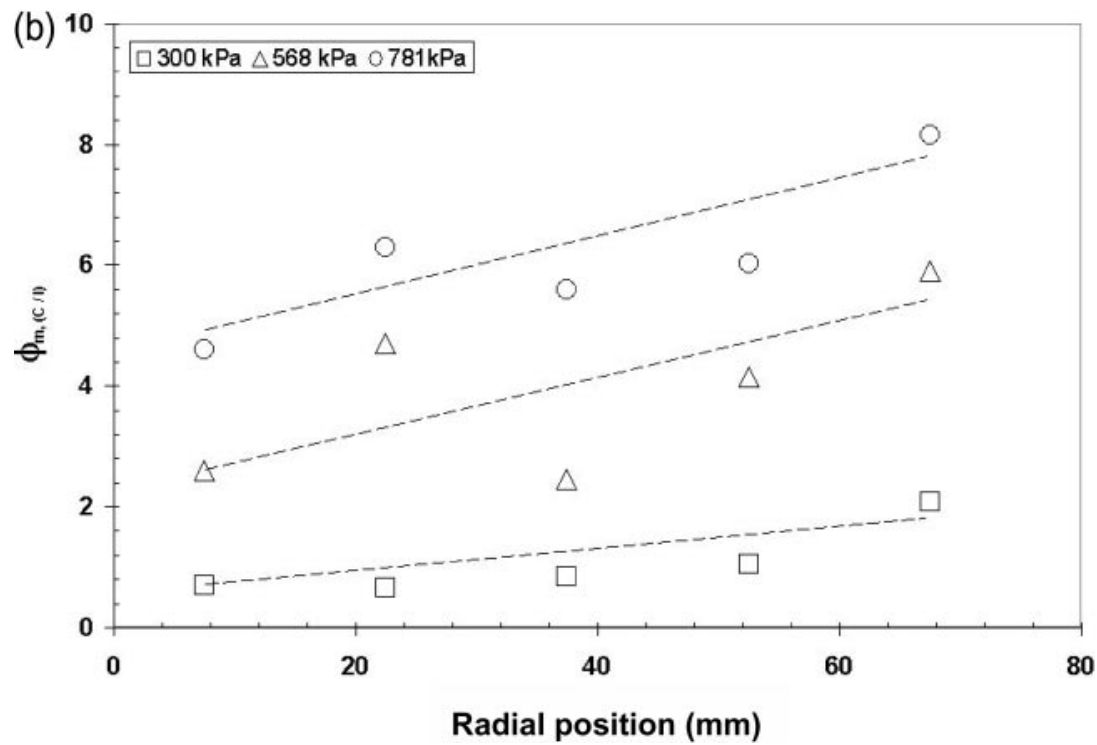

Figure 9. Relative voids shape contributions as a function of radial locations for composites molded at different postfill pressures. (a)Circular/Ellipsoidal; (b) Circular/Irregular.

\section{Effect of Postfill Pressure on Porosity Segregation Through the Specimen Thickness}

As the resin front moves inside the mold, it encounters a heterogeneous porous media (e.g. reinforcement) with highly anisotropic permeability values. Besides this intricate pore network, there are other dissimilar conduits the resin has to flow through during composite molding. For instance, the irregular boundary between the reinforcement and 

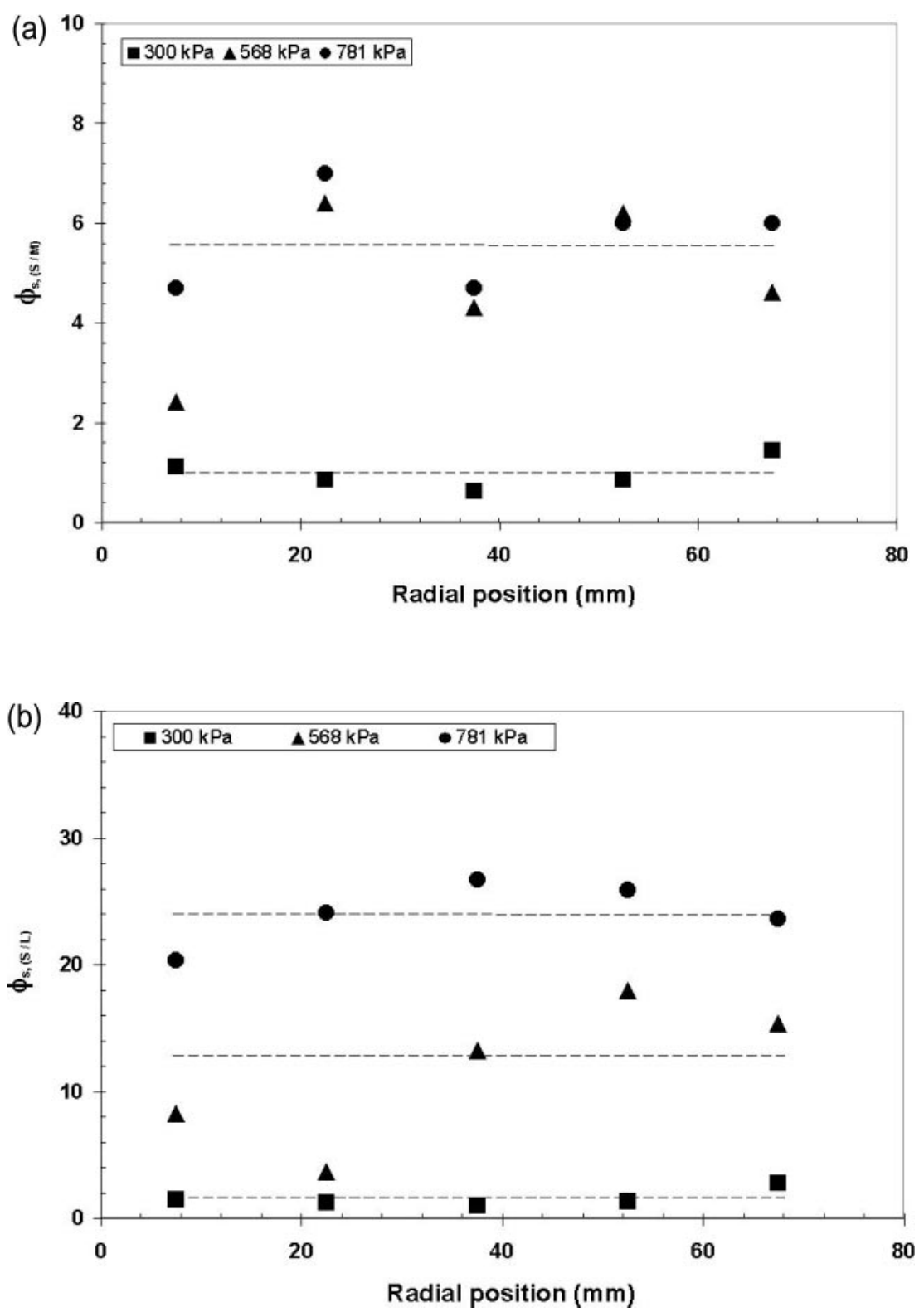

Figure 10. Relative voids size contributions as a function of radial locations for composites molded at different postfill pressures: (a) Small/Medium; (b) Small/Large.

the inner mold surface usually forms gaps (e.g. $1 \mathrm{~mm}$ or less) that can create preferential flow paths leading to a nonuniform impregnation [37]. Voids can form as a result of such inhomogeneous flow field. Therefore, in the current study, microscopic images were taken from the top layer (e.g. area within $1.07 \mathrm{~mm}$ from the top mold wall), bottom layer (e.g. $1.07 \mathrm{~mm}$ from the bottom wall), and from the inner region, to assess the variations in porosity content through the specimen thickness. The total area scanned 


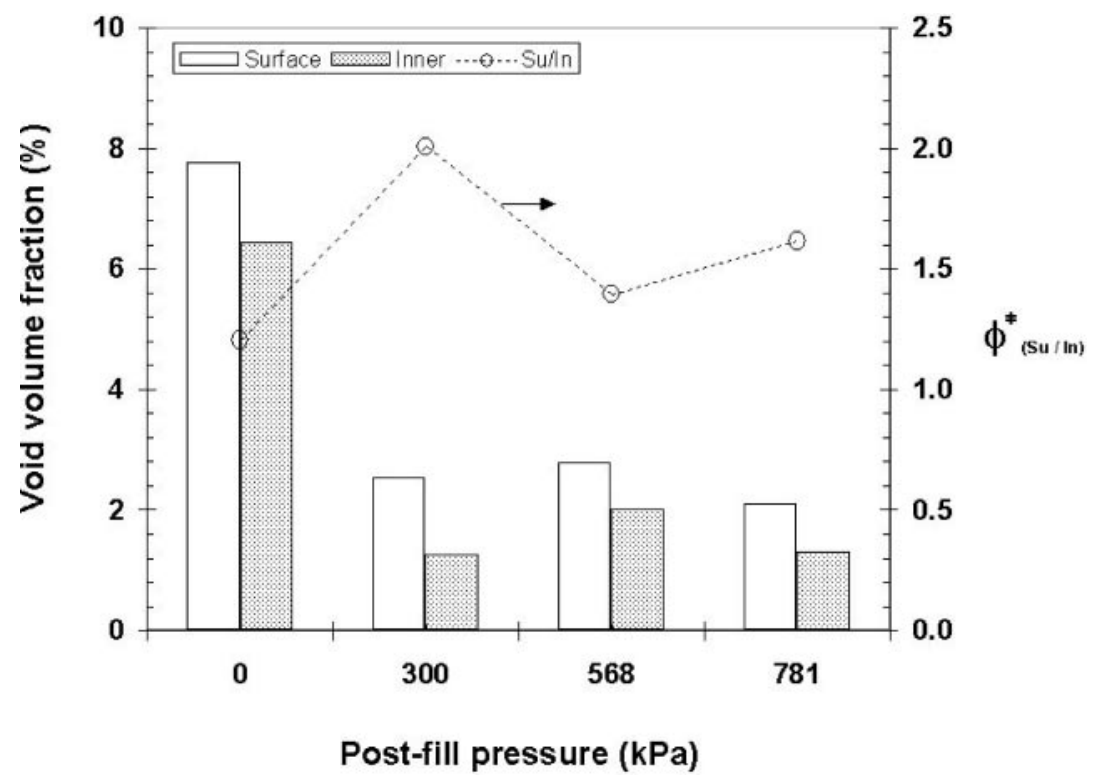

Figure 11. Void volume fraction and relative void presence evaluated at two locations: surface and interior, for composites molded at different postfill pressures.

from the top and bottom layers, which will be referred to as the surface $(\mathrm{Su})$, is numerically equivalent to the area of the inner region (e.g. inner, In). Within these areas, a complete analysis of voids contents, as well as functional variations of voids shape and size, was carried out.

Figure 11 shows results for the percent void volume fraction and relative void areas $\left(\Phi^{*}\right)$ of the surface and inner region of composites molded at different postfill pressures. The first remark that can be made from this data is that the void content in each region follows exactly the same tendency observed previously for the overall void content as a function of the packing level. That is, increased postfill pressures reduce the porosity level both in the part surface as well as in its interior. There is, however, a phenomenon of porosity concentration toward the surfaces in all the composite samples, clearly evidenced from the differences between void fractions measured at the surface and in the interior. Furthermore, comparing the relative void area values, $\Phi^{*}$, it appears as if the void segregation becomes slightly more pronounced with increasing packing pressures. Note for example, that the void area ratio for baseline composites (e.g. $0 \mathrm{kPa}$ ) is very close to one; whereas, at higher postfill pressures this ratio increases, in average, more than $40 \%$ $\left(\Phi^{*}=1.5-2.0\right)$ with respect to that in baseline composites.

Two factors may be considered as potential causes for the observed preferential location of voidage closer to the surface: secondary flow phenomena (e.g. microfountain flow), originated from local velocity variations through the mold cavity spacing; and poor surface energy interaction of the liquid resin with the mold walls. Microstructural variations due to particle segregation are known to occur in particulate-reinforced composites fabricated by injection molding. In a study involving the injection molding of a composite mixture of polystyrene with glass beads of different diameters $(40-75 \mu \mathrm{m}$ up to $500 \mu \mathrm{m})$, Papathanasiou [38] observed a gradual accumulation of the particles towards 
the advancing free surface, as well as an increase of particle concentration at positions further away from the gate. This segregation phenomenon was dependent on the particle size and mold geometry parameters (e.g. size and location of the injection gate). The author explains that if the fountain flow would be operating alone, it would cause the formation of a skin-core structure but could not create an accumulation of particles towards the front surface. Therefore, he proposes an alternative interpretation based on the transverse migration of particles towards the faster-flowing material at the centerplane of the cavity, superimposed to the fountain flow. In the context of our current study, it seems possible to envision a similar situation where voids concentrate at the high-velocity flow front, and are simultaneously pushed towards the walls by the effect of the microfountain transverse flow. Near the walls the resin local velocities are much lower than in the mold midplane; hence, the momentum transfer is greatly reduced and the voids loose all mobility. This mechanism appears to be consistent with the results presented in Figure 12 on the variation of $\Phi^{*}$ with the radial position. Although there is appreciable scatter from a linear fit, the trend line for all data (e.g. with and without postfill pressure) suggests that porosity levels in the surface and in the interior approach each other with increasing distances from the injection gate. Therefore, a plausible explanation for the void segregation phenomena would be that very high front velocities close to the entrance (Figure 3(b)) augment the microfountain flow intensity and more voids are pushed toward the mold surface. As the velocity decreases further away with the radial distance, the front is less affected by the secondary flow phenomena and the driving force for the segregation ceases. The microfountain flow may be more active when

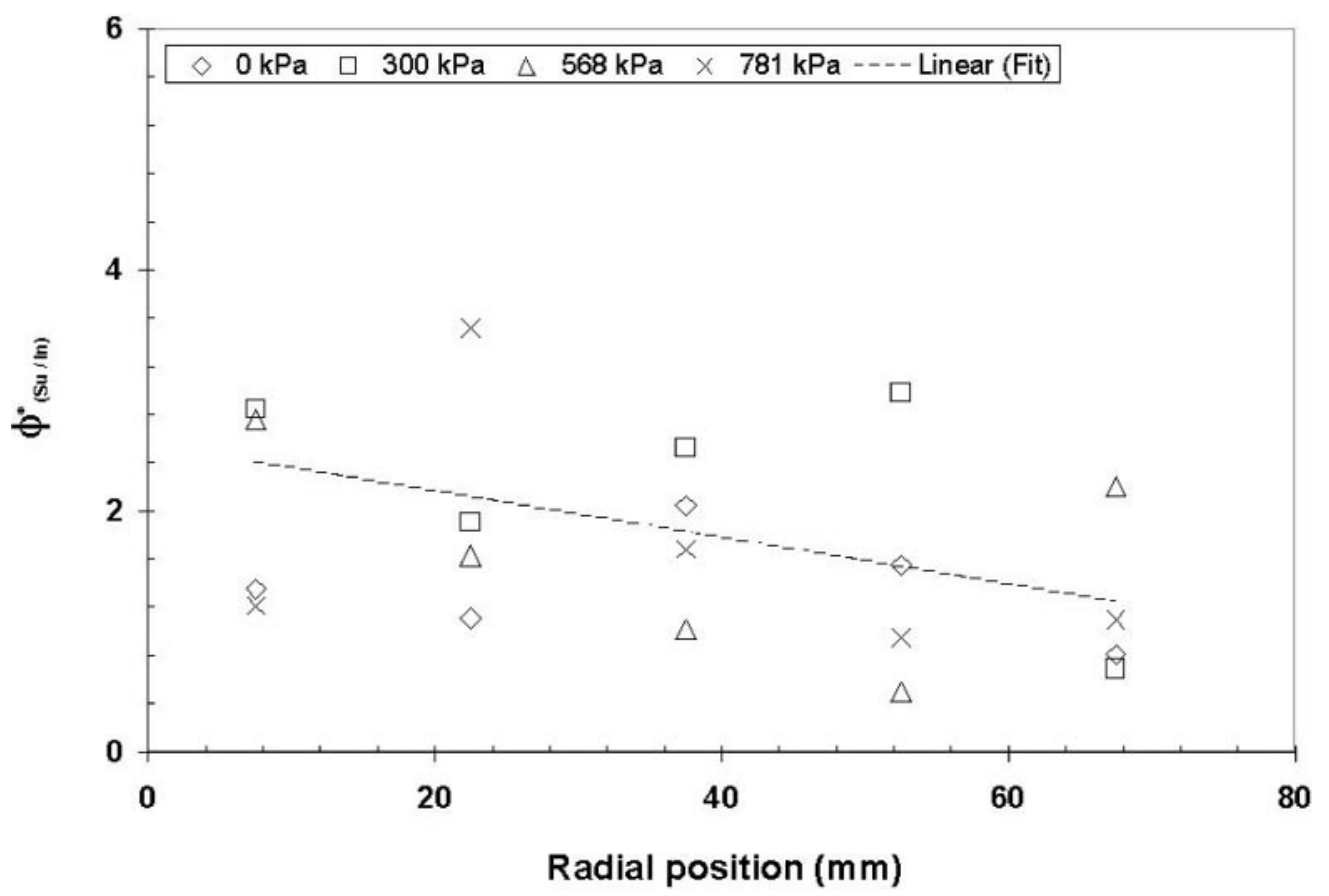

Figure 12. Relative void presence evaluated through the specimen thickness at different radial locations as a function of postfill pressure. 

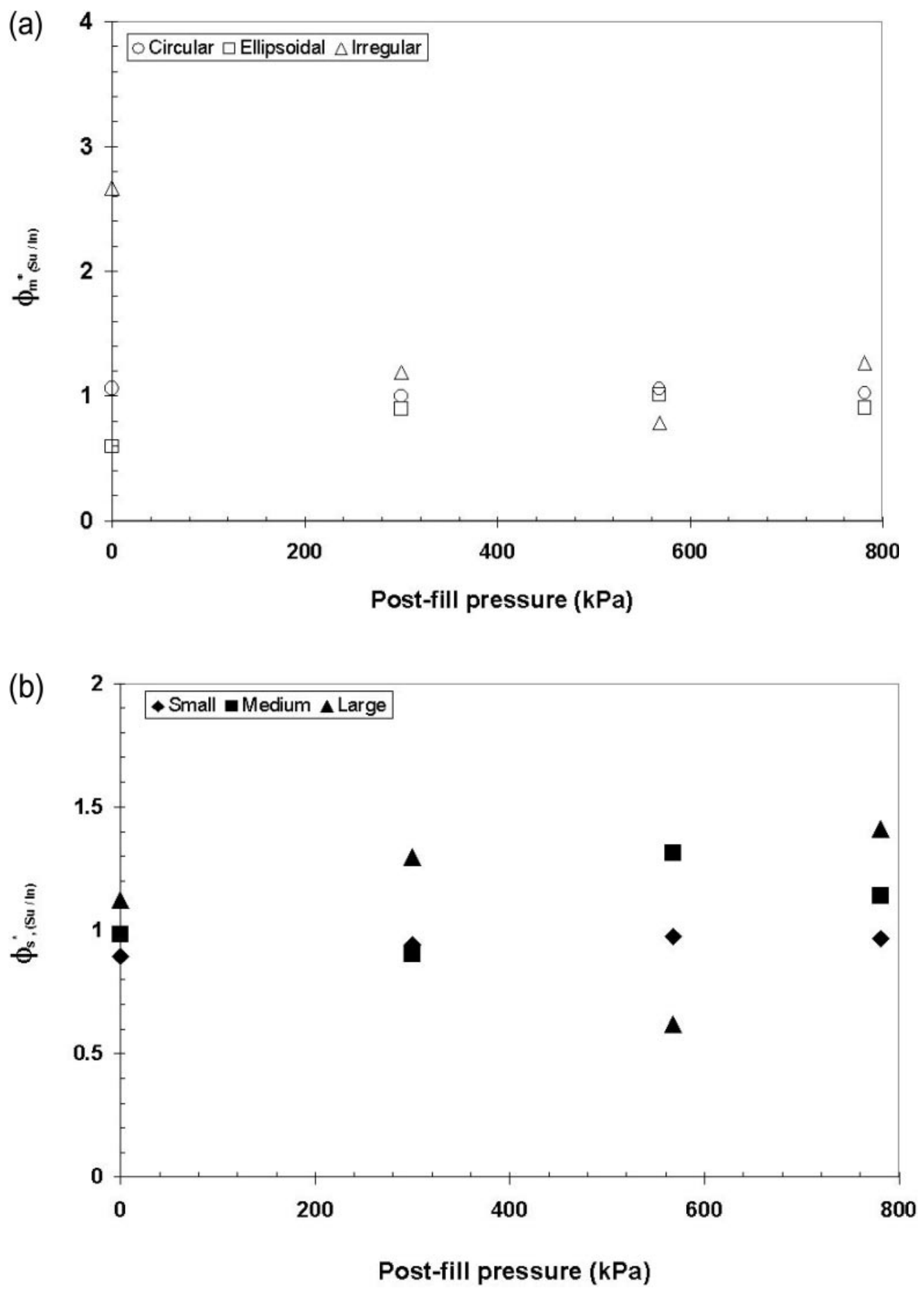

Figure 13. Relative voids shape and size contributions evaluated through the specimen thickness at different postfill pressures: (a) Shape contribution; (b) Size contribution. 
the fiber volume fractions are low, as in the current work, and perhaps loose its effect with a more closely packed tow architecture. This issue has not been investigated in any detail herein, and surely will be a focus of future research efforts. The other possibility for creating a porosity gradient across the composite is related to the poor wettability of the aluminum wall when pretreated with a mold-release agent (e.g. usually composed of Teflon). As listed on Table 1, the surface tension for the impregnating liquid would range between the measured values of $36.3 \mathrm{mN} / \mathrm{m}$, for the polymeric mixture, up to $40.6 \mathrm{mN} / \mathrm{m}$, for the pure resin. Meanwhile, the reported surface tension for the Teflon coating (e.g. polytetrafluoroethylene) is around $23.9 \mathrm{mN} / \mathrm{m}$ at $20^{\circ} \mathrm{C}$ [39]. Therefore, there is an unfavorable wetting condition for the resin when flowing over the coated mold walls, which at high impregnation velocities would favor higher contact angles at the advancing front and greater chances for air entrapment.

Another important observation coming from the through-the-thickness analysis was that void morphometry is also related to the applied postfill pressure level. In Figure 13(a) the parameter, $\Phi_{m}^{*}(\mathrm{Su} / \mathrm{In})$, corresponds to the ratio of number of voids with a particular shape distributed between the surface and the inner regions. As can be seen in this graph, those samples molded without packing pressure tend to accumulate more asymmetrical porosity towards the surface. In contrast, the observed frequency ratios for circular and elliptical voids do not indicate a predominance of these types of voids within the surface region. Howe et al. [16] also found substantially higher proportions of irregular voids at the surface than internally in carbon-fiber composite specimens molded by vacuumassisted RTM. They argued that at the mold surface there is more free space (e.g. between the tows); whereas in the interior part, the tows can intermingle, thus reducing the available free space that resin or voids can occupy. It is well known that voids preferably reside in areas with high permeability [36]. Thus, areas closer to the surface will exhibit higher permeabilities than those in the center of the mold cavity. The availability of free space could perhaps be among the reasons of the similarly higher levels of irregular voidage found in the current study at the surface of composites molded with $0 \mathrm{kPa}$ postfill pressure. However, a direct comparison with Howe's study may be deemed inappropriate as the void formation and void transport mechanisms are certainly different for vacuumassisted molding. As depicted by Figure 13(b), the applied postfill pressure also causes a slight drop in the amount of both inner and surface voids with large and medium sizes, with apparently higher reductions taking place for the inner large voids $\left(\Phi_{s}^{*}=1.5\right)$ at the maximum postfill pressure (e.g. $781 \mathrm{kPa})$.

\section{CONCLUSIONS}

The mechanical performance of fiber-reinforced composites is strongly linked to the adhesive strength of their interfaces, as well as to the presence of process-induced defects such as voids and dry areas. To date, there is no commercial method capable of manufacturing completely void-free parts. The existing alternatives to reduce the porosity, either incur high operational costs, or have not been successful at all. In this paper, we have conducted an assessment on the extent of porosity reduction that can be attained when different postfill pressure levels are applied to the mold after the filling has been completed. The first set of results includes the effect of flow front velocities on the formation of voids when postfill pressure is not applied. Previous data [13], obtained in an RTM setup for low-speed processing (e.g. flow rate $0.2 \mathrm{~cm}^{3} / \mathrm{s}$ ), was compared with the 
current molding procedure (e.g. high-speed processing) that was capable of injecting at flow rates in the order of $5.32 \mathrm{~cm}^{3} / \mathrm{s}$. A tenfold increase in the overall void content takes place in the composites molded at high-speed compared to the same type of parts molded at lower volume flow rates. Furthermore, overall void area fractions in the composites molded with both processing schemes were found to be a function of the modified capillary numbers and flow front velocities, in accordance with data presented in the literature for model fluids.

Transient pressure reading at the injection port revealed that, both the filling operation as well as the establishment of a final postfill pressure was adequately controlled with the current molding setup and molding procedures. The major effect observed by applying the extra packing was a reduction of more than $75 \%$ in overall porosity from the baseline level (e.g. $0 \mathrm{kPa}$ ) for composites molded with the short fill time setup. The application of pressure posterior to the filling induced void migration along the radius of the parts, which is believed to be the result of several transport mechanisms represented by shearing forces and void compression and dissolution. This behavior contrasts with that encountered in composites molded without packing, where the porosity preferentially concentrated in the center of the parts. The postfill pressure also seemed to have a strong influence on the shape and size of the voids. Basically, the overall content of irregular voids is substantially reduced with increasing levels of pressure. The same holds true for both the elliptical and circular voids, which are almost completely eliminated as the packing pressure increases. It is worth noting that void morphology also changed as a function of radial position, with increasing proportions of circular voids being located towards the vent openings.

In terms of the void size, the most striking observation was that small voids augment their relative presence with respect to the medium and large voidage as the postfill pressure increases. This is consistent with the disruption of the pressure balance between the air pressure inside the void and the pressure surrounding the void (e.g. postfill pressure). Finally, the high-velocity impregnation front seems to promote the onset of secondary flow phenomena inside the mold cavity, such as the microfountain flow near the mold surface, which is perhaps responsible for the higher porosity levels recorded close to the composites surface. In all, these results have practical implications for the composites industry in the sense that high-speed RTM molding may be used to produce parts with negligible voidage by applying postfill pressures.

\section{REFERENCES}

1. Johnston, N.J. (2000). Aerospace Materials and Processes for the Next Decade, In: Book of Abstracts, Composites 2000: An International Symposium on Composite Materials, University of Delaware.

2. Fong, L. and Advani, S. (1995). In: Resin Transfer Molding - Technical Report CCM 95-09, University of Delaware Center for Composite Materials.

3. Roychowdhury, S. (1995). Void Formation and Growth in Amorphous Thermoplastic Polymeric Materials, PhD Thesis, University of Delaware, Newark, DE.

4. Judd, N.C.W. and Wright, W.W. (1978). Voids and Their Effects on the Mechanical Properties of Composites - An Appraisal, SAMPE J., 14(1): 10-14.

5. Thomason, J.L. (1995). The Interface Region in Glass Fiber-Reinforced Epoxy Resin Composites: 1. Sample Preparation, Void Content and Interfacial Strength, Composites, 26(7): 467-475. 
6. Patel, N., Rohatgi, V. and Lee, J.L. (1995). Micro Scale Flow Behavior and Void Formation Mechanism During Impregnation Through a Unidirectional Stitched Fiberglass Mat, Polymer Engineering and Science, 35(10): 837-851.

7. Patel, N. and Lee, J.L. (1996). Modeling of Void Formation and Removal in Liquid Composite Molding. Part II: Model Development and Implementation, Polymer Composites, 17(1): 104-114.

8. Young, W.B. (1996). The Effect of Surface Tension on Tow Impregnation of Unidirectional Preform in Resin Transfer Molding, Journal of Composite Materials, 30(11): 1191-1209.

9. Chen, Y.-T., Davis, H.T. and Macosko, C.W. (1995). Wetting of Fiber Mats for Composites Manufacturing: I. Visualization Experiments, AICHE J., 41(10): 2261-2273.

10. Lundström, T.S. and Gebart, B.R. (1994). Influence From Process Parameters on Void Formation in Resin Transfer Molding, Polymer Composites, 15(1): 25-33.

11. Blackmore, B., Li, D. and Gao, J. (2001). Detachment of Bubbles in Slit Microchannels by Shearing Flow, Journal of Colloid and Interface Science, 241(2): 514-520.

12. Varna, J., Joffe, R., Berglund, L. and Lunström, T.S. (1995). Effect of Voids on Failure Mechanisms in RTM Laminates, Composites Science and Technology, 53(2): 241-249.

13. Olivero, K.A., Barraza, H.J., O'Rear, E. and Altan, M.C. (2002). Effect of Injection Rate and Post-Fill Cure Pressure on Properties of Resin Transfer Molded Disks, Journal of Composite Materials, 36(16): 2011-2028.

14. Mahale, A.D., Prud'homme, R.K. and Rebenfeld, L. (1992). Quantitative Measurement of Voids Formed During Liquid Impregnation of Nonwoven Multifilament Glass Networks Using an Optical Visualization Technique Polymer Engineering and Science, 32(5): 319-326.

15. Ghiorse, S.R. (1993). Effect of Void Content on the Mechanical Properties of Carbon/Epoxy Laminates, SAMPE Quarterly, 24(2): 54-59.

16. Howe, C.A., Paton, R.J. and Goodwin, A.A. (1997). A Comparison Between Voids in RTM and Prepreg Carbon/Epoxy Laminates. In: Proceedings of ICCM-11, 4: 46-51.

17. Wisnom, M.R., Reynolds, T. and Gwilliam, N. (1996). Reduction in Interlaminar Shear Strength by Discrete and Distributed Voids, Composites Science and Technology, 56(1): 93-101.

18. Gebart, R. and Strömbeck, L. (2000). Principles of Liquid Composite Molding. In: Davé, R.S. and Loos, A.C. (eds), Processing of Composites, pp. 358-387, Hanser Publishers, Munich.

19. Shamsuddin, I., Siegel, M.C., Sadler, R.L. and Avva, V.S. (1995). Effect of Surface-Active Agents on Void Minimization in RTM Processing of Carbon-Epoxy Composites. In: International SAMPE Technical Conference, 27: 457-471.

20. Stabler, W.R., Tatterson, G.B., Sadler, R.L. and El-Shiekh, A.H.M. (1992). Void Minimization in the Manufacture of Carbon Fiber Composites by Resin Transfer Molding, SAMPE Quarterly, 23(2): 38-42.

21. Pantelelis, N.G., Bikas, A. and Kanarachos, A.E. (2000). Vibration Assisted Resin Transfer Moulding (VIARTM): A New Technique to Improve RTM Performance and Part Quality. In: Annual Technical Conference-Society of Plastics Engineers, 58(2): 2351-2355.

22. Nowak, T. and Chun, J.H. (1993). Optical Measurements of Preform Impregnation in Resin Transfer Molding. In: Materials Research Society Symposium Proceedings, 305: 241-246.

23. Strömbeck, A. (1993). Optimization of the Resin Transfer Molding Process for Advanced Composites. In: Proceedings of the 9th International Conference of Composite Materials, 3: 497-504.

24. Lee, C.H. and Wei, K.H. (2000). Effect of Material and Process Variables on the Performance of Resin-Transfer-Molded Epoxy Fabric Composites, Journal of Applied Polymer Science, 77(10): 2149-2155.

25. Lee, C.H. and Wei, K.H. (2000). Resin Transfer Molding Process (RTM) of a High Performance Epoxy Resin. II: Effects of Process Variables on the Physical, Static and Dynamic Mechanical Behavior, Polymer Engineering Science, 40(4): 935-943.

26. Larson, B. and Drzal, L. (1994). Glass Fibre Sizing/Matrix Interphase Formation in Liquid Composite Moulding: Effects on Fibre/Matrix Adhesion and Mechanical Properties, Composites, 25(7): 711-721. 
27. Chui, W.K., Glimm, J., Tagerman, F.M., Jardine, A., Madsen, J.S., Donellan, T. and Leek, R. (1997). Process Modeling in Resin Transfer Molding as a Method to Enhance Product Quality, SIAM Review, 39(4): 714-727.

28. Barraza, H.J., Hwa, M.J., Blakely, K., O’Rear, E.A. and Grady, B.P. (2001). Wetting Behavior of Elastomer-Modified Glass Fibers, Langmuir, 17(17): 5288-5296.

29. Kang, M.K., Lee, W.I. and Hahn, H.T. (2000). Formation of Microvoids During Resin-Transfer Molding Process, Composites Science and Technology, 60: 2427-2434.

30. Ghiorse, S.R. (1991). A Comparison of Void Measurement Methods for Carbon/Epoxy Composites, U. S. Army Materials Technology Laboratory, Report MTL TR 91-13.

31. Russ, J. (1986). Practical Stereology, 1st edn, Plenum Press, New York.

32. Chan, A.W., Larive, D.E. and Morgan, R.J. (1993). Anisotropic Permeability of Fiber Preforms: Constant Flow Rate Measurement, Journal of Composite Materials, 27(10): 996-1008.

33. Palmese, G.R. and Karbhari, V.M. (1995). Effects of Sizings on Microscopic Flow in Resin Transfer Molding, Polymer Composites, 16(4): 313-318.

34. Rohatgi, V., Patel, N. and Lee, J. (1996). Experimental Investigation of Flow-Induced Microvoids During Impregnation of Unidirectional Stitched Fiberglass Mat, Polymer Composites, 17(2): 161-170.

35. Lundström, T.S. (1997). Measurement of Void Collapse During Resin Transfer Moulding, Composites Part A, 28A: 201-214.

36. Lundström, T.S. (1996). Bubble Transport Through Constricted Capillary Tubes with Application to Resin Transfer Molding, Polymer Composites, 17(6): 770-779.

37. Ambrosi, D., Farina, A. and Preziosi, L. (2002). Recent Developments and Open Problems in Composite Materials Manufacturing. In: Anile, A.M., Capasso, V. and Greco, V. (eds), Progress in Industrial Mathematics at ECMI 2000, Springer-Verlag, Berlin.

38. Papathanasiou, T.D. (1996). Microstructure Evaluation During Moulding of ParticulateReinforced Thermoplastic Composites, International Polymer Processing, 11(3): 275-283.

39. Sperling, L.H. (1992). Introduction to Physical Polymer Science, 2nd edn, John Wiley \& Sons, New York. 